\title{
An Investigation of LED Street Lighting's Impact on Sky Glow
}

April 2017

Prepared for:

Solid-State Lighting Program Building Technologies Office Office of Energy Efficiency and Renewable Energy

U.S. Department of Energy

Prepared by:

Pacific Northwest National Laboratory 


\section{An Investigation of LED Street Lighting's Impact on Sky Glow}

Prepared in support of the DOE Solid-State Lighting Technology Program

Study Participants:

Pacific Northwest National Laboratory

U.S. Department of Energy

Slovak Academy of Sciences

Cégep de Sherbrooke

Interdisciplinary Center for Metropolitan Studies

Bruce Kinzey

Tess E. Perrin

Naomi J. Miller

Miroslav Kocifaj

Martin Aubé

Héctor S. Lamphar

April 2017

Prepared for:

U.S. Department of Energy

under Contract DE-AC05-76RL01830

Pacific Northwest National Laboratory

Richland, Washington 99352 
PNNL-26411

\title{
DISCLAIMER
}

This report was prepared as an account of work sponsored by an agency of the United States Government. Neither the United States Government nor any agency thereof, nor Battelle Memorial Institute, nor any of their employees, makes any warranty, express or implied, or assumes any legal liability or responsibility for the accuracy, completeness, or usefulness of any information, apparatus, product, or process disclosed, or represents that its use would not infringe privately owned rights. Reference herein to any specific commercial product, process, or service by trade name, trademark, manufacturer, or otherwise does not necessarily constitute or imply its endorsement, recommendation, or favoring by the United States Government or any agency thereof, or Battelle Memorial Institute. The views and opinions of authors expressed herein do not necessarily state or reflect those of the United States Government or any agency thereof.

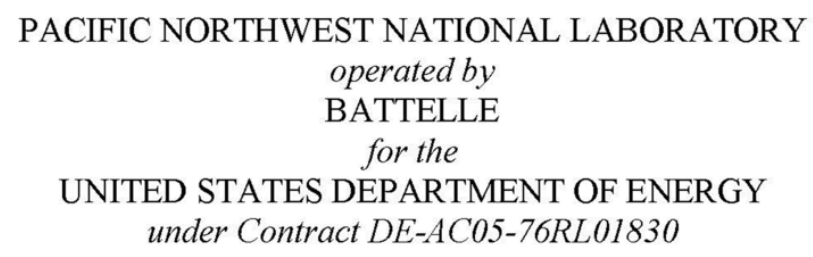

Printed in the United States of America

Available to DOE and DOE contractors from the

Office of Scientific and Technical Information,

P.O. Box 62, Oak Ridge, TN 37831-0062;

ph: (865) 576-8401

fax: $(865) 576-5728$

email: reports@adonis.osti.gov

\author{
Available to the public from the National Technical Information Service \\ 5301 Shawnee Rd., Alexandria, VA 22312 \\ ph: (800) 553-NTIS (6847) \\ email: orders@ntis.gov $<$ http://www.ntis.gov/about/form.aspx> \\ Online ordering: http://www.ntis.gov
}

This document was printed on recycled paper. 


\section{Acknowledgements}

This project would not have been possible without the full collaboration and cooperation of the following individuals, whose support is gratefully acknowledged:

- Tim Carlson, Principal IT Architect at PNNL, facilitated the utilization of the PNNL Institutional Computing (PIC) program's resources. ${ }^{1}$ These resources included assistance and guidance writing scripts to execute the SkyGlow Simulator model along with the use of a supercomputer, Constance, and ultimately a Windows HPC cluster, housed at PNNL's headquarters in Richland, WA.

- Ian Ashdown, President at by Heart Consultants, contributed an early model SkyGlowCalc and shared a significant amount of time and effort investigating not only the numerous models available but also the literature discussing the various impacts to sky glow.

- Marc Ledbetter, Program Manager of PNNL's Solid-State Lighting, provided direction, insight, and numerous hours of peer review.

- Jim Brodrick, Lighting Program Manager for the U.S. Department of Energy in the Building Technologies Office, supported this project and provided unending patience.

1 PIC is part of the Lab's computing strategy to nurture a culture of computational science by offering cost-effective services and hardware capabilities. For more information about the PIC program, please see the PIC Resources page:

http://pic.pnnl.gov/resources.stm. 


\section{Executive Summary}

Like all broad-spectrum (i.e., white) light sources, light-emitting diodes (LEDs) produce a portion of their output in shorter wavelengths, which may include varying components of violet, blue, cyan, and other colors. Because shorter wavelengths scatter more readily in the Earth's atmosphere than longer wavelengths, like yellow and red, and because of certain biological sensitivities to shorter wavelengths, a variety of concerns have been raised regarding the potential impact from converting exterior lighting sources with low, short wavelength content, primarily high-pressure sodium (HPS), to broad-spectrum LED. Street lighting is often a focus of these concerns, including potential reduction of night-sky visibility from greater levels of scattered light, commonly referred to as sky glow, and potential health issues from exposure to those higher levels of light. This document focuses on potential changes to sky glow that can be expected from LED street lighting conversions, and does not address health concerns.

Multiple factors contribute to the ultimate levels of sky glow in any location. A major consideration is that street lighting, the focus of this document, is only one of many sources of light at night in urban areas. Other sources include building interior lights escaping from exterior windows, architectural and landscape lighting, signage, parking lots and garages, recreational lighting, and vehicular lighting. The findings in this document consequently represent only the contributions to sky glow from the street lighting system and may not even address the primary sources of sky glow in locations such as a large urban area. The results and related undertakings planned in any real location should be considered in that context.

While increasing the short wavelength content of exterior lighting sources increases the potential for sky glow, other characteristics of LED street lighting luminaires can reduce or completely offset these effects. The three main characteristics of luminaires that influence sky glow are spectral power distribution (SPD), total lumen output, and luminaire light distribution (and, most importantly, the amount of that distribution emitted as uplight). Each of these characteristics can be specified through the selection of luminaires and should therefore be carefully evaluated as part of the system design.

External factors also influence how much sky glow a given observer sees, such as the observer's location relative to the source of the light (especially their distance from it), the size of the source (i.e., city area), the atmospheric conditions at the time of observation, the heights of poles, surrounding geographical features, and the reflectance of the ground surface. This project has estimated the influence of the first three factors using a model well known throughout the astronomical community, SkyGlow Simulator, developed by Miroslav Kocifaj. ${ }^{2}$ The influence of each factor was calculated independently during an extended set of computer runs (approximately 200,000). Generalized assumptions were necessary even with this large number of runs to prevent the computation effort from growing even larger, as were significant simplifications to such complex topics as atmospheric aerosol concentration, composition, and physical characterization (i.e., particle size, shape, and distribution).

As in any modeling effort, the results are only as valid as their underlying assumptions, so care was taken to select values for variables that are reasonable and representative of typical conditions and scenarios. The latter

2 Miroslav Kocifaj is a senior researcher at the ICA Institute of the Slovak Academy of Sciences. 
is key to interpreting the results contained in this report. While any number of hypothetical constructs could be explored, such as updated versions of incumbent technologies (for example, HPS with improved optical control) or "engineered" SPDs designed to optimize specific properties of a light source, the primary focus of this investigation is to examine representative street lighting situations that exist across the U.S. today, both the incumbent systems and the LED systems replacing them. The results are thereby intended to represent what is happening in the U.S. rather than what could happen if the study were free to invent alternative characteristics of future street lighting systems.

In a few cases, values were selected because they are commonly used in other atmospheric modeling efforts (such as a uniform ground reflectivity of $15 \%)$. In terms of luminaire characteristics, four levels of uplight $(0 \%$, $2 \%, 5 \%$, and $10 \%$ ) were compared, along with two percentages of lumen output ( $100 \%$ and $50 \%$ ). The latter were chosen to show the relative influence of lumen output instead of absolute lumen values, given the wide variation of product outputs within even a single installation and the intent of this study to be more generic in its findings. Finally, 10 different SPDs from actual products of different light source types and correlated color temperatures (CCTs) were modeled, along with a hypothetical source whose radiant energy output is equal across the visible spectrum. The equal energy spectrum was included specifically to investigate the influence of individual wavelength bands on sky glow, in $5 \mathrm{~nm}$ increments from 380 to $780 \mathrm{~nm}$, to identify which wavelengths contribute more to sky glow under different conditions.

The model calculates sky glow impacts from street lighting in terms of diffuse irradiance $\left(\mathrm{W} / \mathrm{m}^{2}\right)$ or illuminance (scotopic-cd $/ \mathrm{m}^{2} / \mathrm{sr}$ or scotopic-lux) on the horizontal plane, incident upon an observer for the entire night sky dome. To facilitate comparisons, these results are then reported relative to the baseline conditions as multiples or fractions thereof. Results are reported both as unweighted sky glow, which reflect the raw radiant power of the sky glow incident upon the observation point, and scotopically weighted sky glow to better account for its visual impact on human observation of the night sky.

Ultimately, sky glow is not determined by any single factor but by the combination of factors present in any given situation. Figures ES.1 and ES.2 show a progression of effects from successively combining the different factors modeled, dividing the results into sky glow impacts for the "near" observer located at the edge of the city (Figure ES.1) and for the "distant" observer located $40 \mathrm{~km}$ from the city center (Figure ES.2). Each graphic is further subdivided by unweighted or scotopically weighted results, by atmospheric condition (ATM1-4 representing a range from clear to increasingly turbid atmospheres, ATM5 representing complete cloud cover), and finally by the SPD of the source. The charts also show the sky glow results for an HPS incumbent (displayed as the red dashed line set at 1.0), but do not show results from two additional incumbent sources that were modeled and are contained in the main body of this report, a $4000 \mathrm{~K}$ metal halide source and a low-pressure sodium source, nor do they include the hypothetical equal energy spectrum.

The results are displayed in three progressive tiers: the top chart shows the isolated effect of replacing the baseline HPS SPD with the various other LED SPDs modeled with no other modifications; the middle chart adds the effect of reducing luminaire lumen output by half compared to the baseline HPS (a typical result for conversions in the U.S.); and the bottom chart further adds the impact of eliminating uplight from the luminaires, assuming a typical HPS baseline value of $2 \%$. The bottom charts in both figures thereby represent typical conversion scenarios in the U.S.-an incumbent HPS cobra head product with $2 \%$ uplight replaced by each of the LED products listed, at half the light output and $0 \%$ uplight. Note that the values represent static conditions, and so do not include any additional reductions possible through the use of adaptive lighting (e.g., dimming). Such impacts could be significant because contributions to sky glow scale directly with light output. 

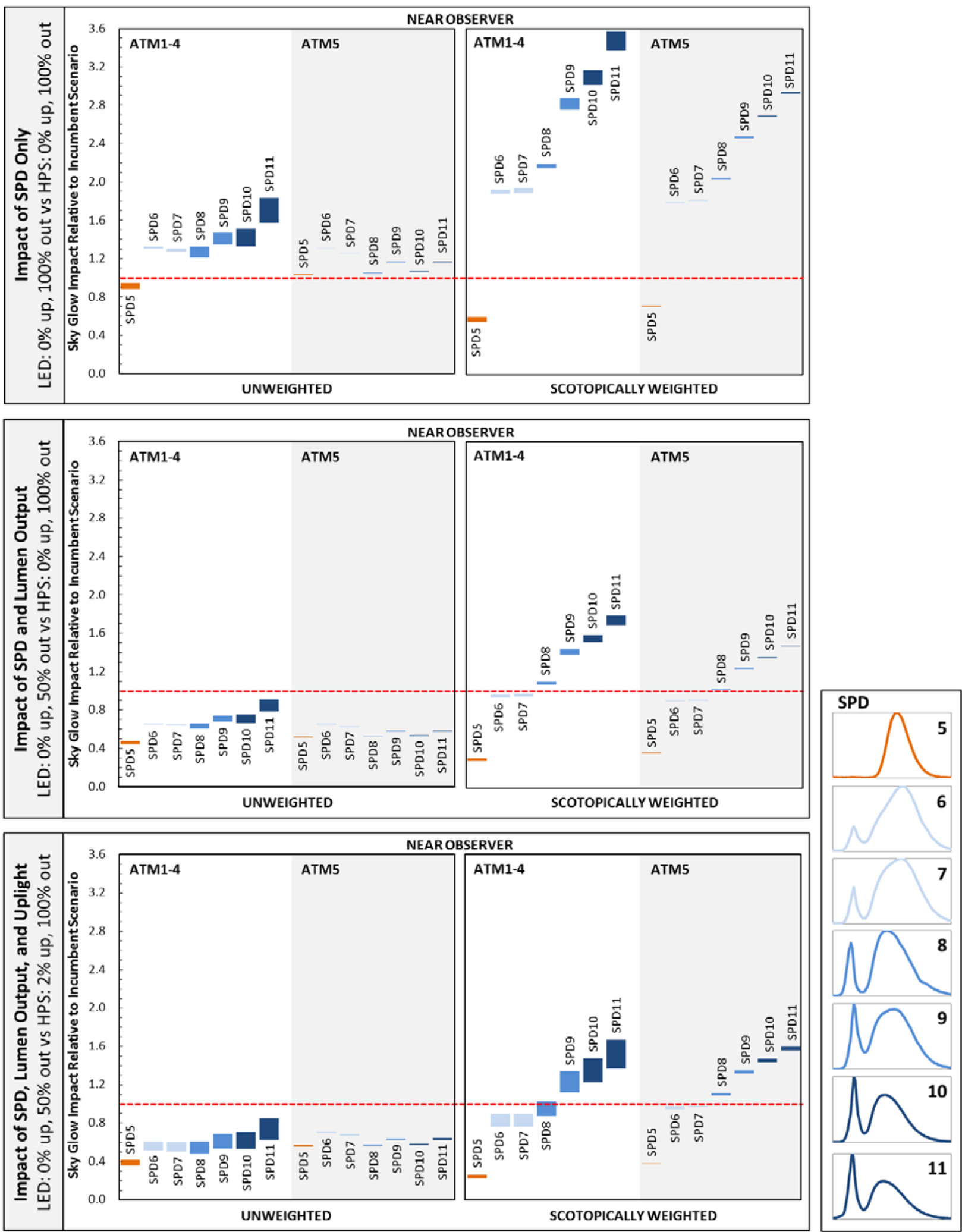

Figure ES.1 Results of the LED street lighting conversions, from the near observer position at the edge of the city. Each plot shows the relative skyglow irradiance contribution from street lights compared to the HPS baseline. The top chart shows the isolated effect of replacing the baseline HPS SPD with that of the various other SPDs modeled; the middle chart adds the effect of reducing luminaire output by half compared to the baseline HPS; the bottom chart further adds the impact of eliminating uplight from the luminaires, assuming a typical HPS baseline value of $2 \%$ (note this represents an increase from the first two charts using $0 \%$ ). The baseline is represented by the dashed red line at the normalized value of 1.0. 

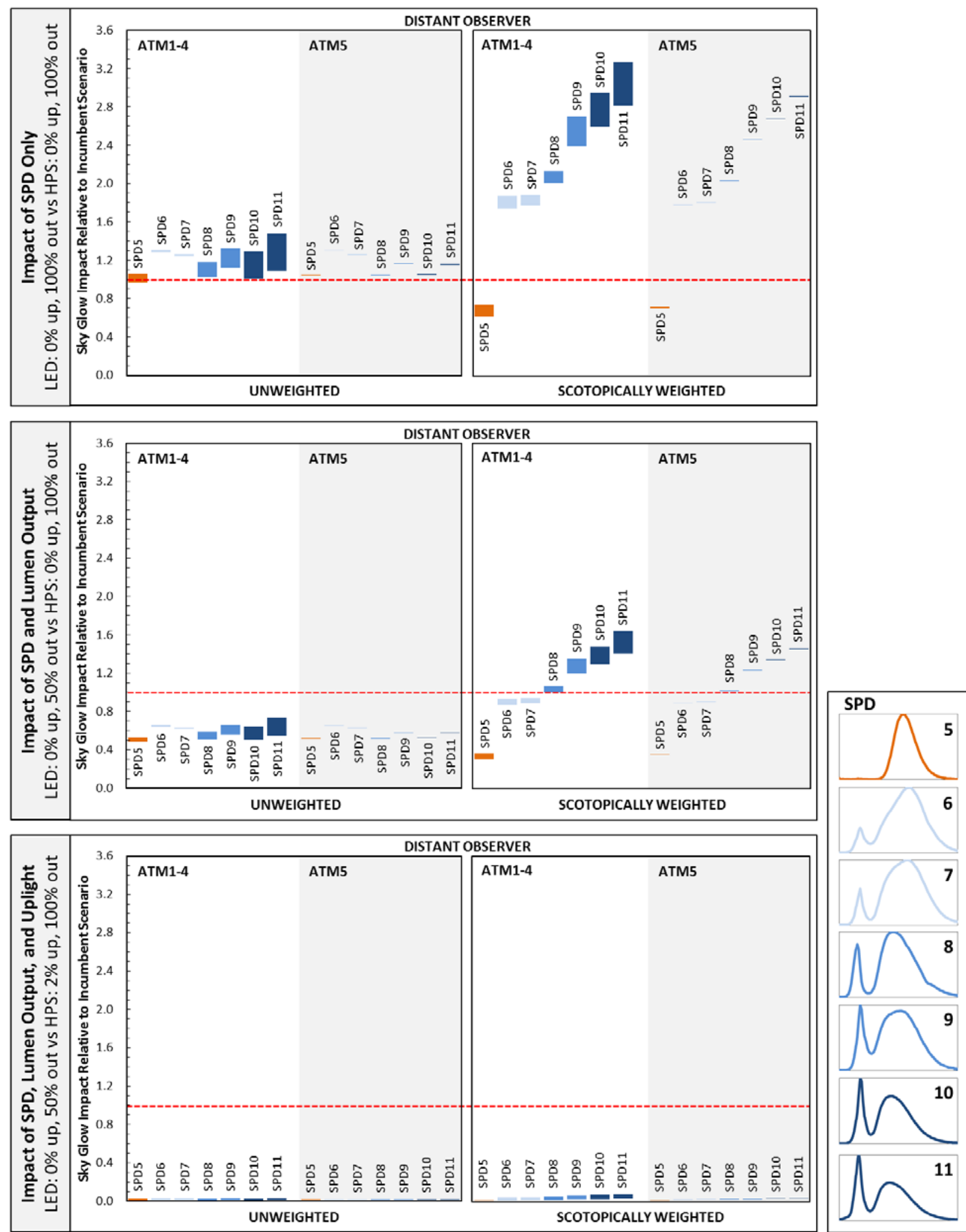

Figure ES.2 Results of the LED street lighting conversions, from the distant observer position $\mathbf{4 0} \mathrm{km}$ from city center. Each plot shows the relative skyglow irradiance contribution from street lights compared to the HPS baseline. The top chart shows the isolated effect of replacing the baseline HPS SPD with that of the various other SPDs modeled; the middle chart adds the effect of reducing luminaire output by half compared to the baseline HPS; the bottom chart further adds the impact of eliminating uplight from the luminaires, assuming a typical HPS baseline value of $2 \%$ (note this represents an increase from the first two charts using $0 \%$ ). The baseline is represented by the dashed red line at the normalized value of 1.0. 
A key finding is that, in a typical U.S. conversion, all of the LED products reduce sky glow when the results are unweighted, for both the near and distant observers. Each bar in the graphs depicts the range of possible sky glow impact, from minimum to maximum, given the fixed conditions noted (atmospheric condition [ATMX], source SPD, and weighting), while allowing all other variables to float within their defined ranges. When the results are instead scotopically weighted to evaluate the effects on human vision (i.e., visibility of the night sky), some LED products reduce sky glow for the near observer and others increase it, compared to the baseline. Another important finding evident in the modeling results is that CCT is not a very reliable indicator of sky glow impacts, especially when scotopic weighting is not applied (evident in the irregular and overlapping performance of the SPDs when ordered by increasing CCT). The overall range of results for LED luminaires across most conditions for the near observer is from about 0.4 to 0.8 times the unweighted baseline HPS sky glow, and 0.2 to 1.6 times the scotopically weighted baseline HPS sky glow. For the distant observer, even at only $40 \mathrm{~km}$ (about 25 miles) from the city center, the elimination of uplight from the luminaires has nearly removed (by $95 \%$ or more) the contribution to sky glow from the street lighting system, for both the unweighted and scotopically weighted results, under all atmospheric conditions. It should also be noted here, however, that this modeling effort did not consider effects of obstruction by buildings or terrain; in locations where such obstructions already block low-angle uplight, elimination of uplight from the luminaires will not have as dramatic an effect.

Except for installations made in the early years of LED street lighting conversions (roughly prior to 2010), most to date have involved $4000 \mathrm{~K}$ CCT products. (Beginning in 2016, some sites also started considering or installing $3000 \mathrm{~K}$ products in their conversion projects.) Although this cannot be confirmed without analyzing the specific SPDs for the new LED systems, this study suggests that, for residents near the city, the visible contribution to sky glow from a typical street light conversion (i.e., half the output; $0 \%$ uplight) should be no worse than before, and has possibly improved. Unweighted results are more relevant to astronomical instrument observation, so when the modeled conditions are achieved, sky glow from the street lights should be considerably reduced (by roughly one-third to one-half) for any observatories near the city.

Distant from the city, the sky glow contribution from the typical LED street lighting conversion appears to be negligible, at all SPDs, for human observers (e.g., scotopically weighted results) as well as astronomical equipment (e.g., unweighted results). Although the reduction is striking even at this relatively short distance of $40 \mathrm{~km}$, street lighting must again be considered in the context of accounting for only one (now former) source of sky glow from an urban area, as the others remain unaffected.

Near the city, the sky glow contributions from the street lighting system can be further reduced by substituting an LED product with less short wavelength content, as often (though not always) found in lower CCT options, or, as noted, by decreasing the light output through dimming or by eliminating unneeded lights. The designer may want to consider the additional value of pursuing such reductions in the context of the overall system design. These include determining just how much light is needed in a given location and how those needs potentially change over the course of the evening-identifying where light is needed, and just as importantly, where it is not, and selecting the color characteristics of the source that are most appropriate to the specific use (and possibly, how they may change over the course of the evening as well).

The artistry in lighting design is in achieving the optimal balance not only among these elements, but also in accommodating safety and economic considerations, aesthetics, user preference, and any additional issues that must be taken into account. The wide flexibility of LED sources provides many choices to the system designer for pursuing the optimal balance for any given set of circumstances. 


\section{Contents}

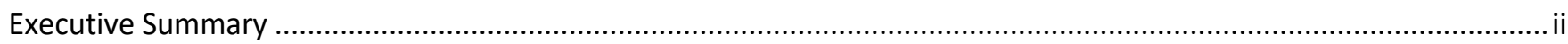

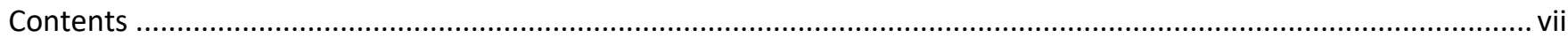

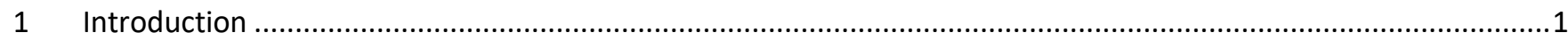

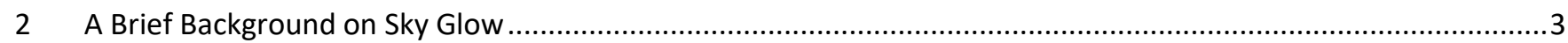

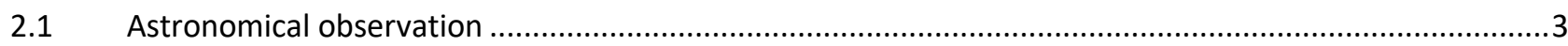

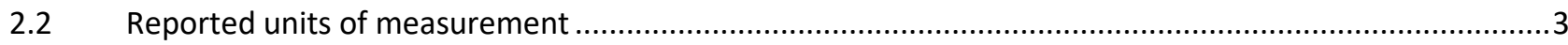

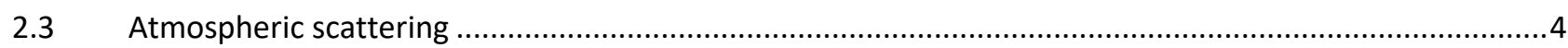

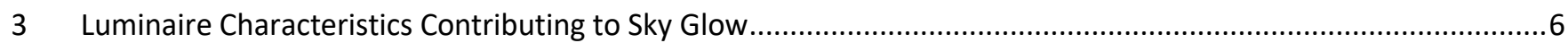

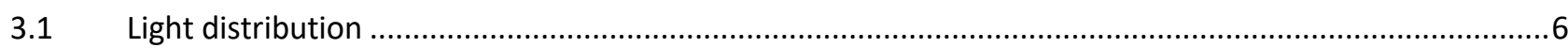

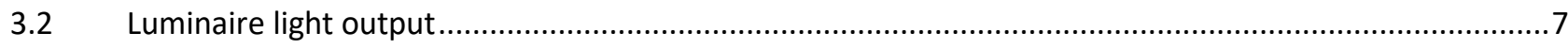

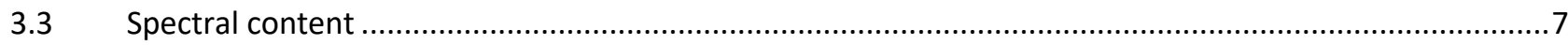

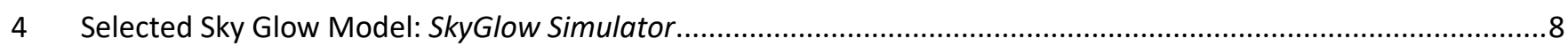

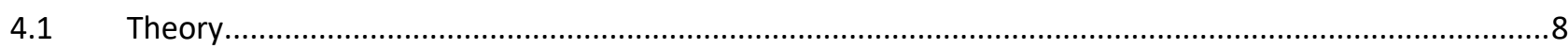

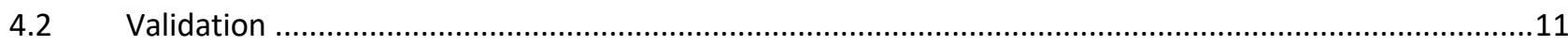

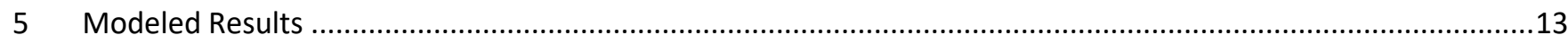

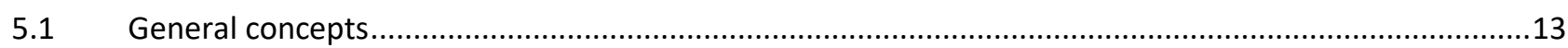

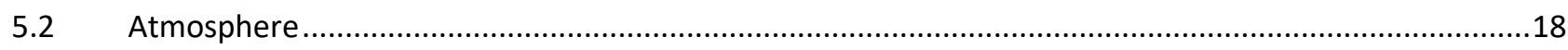

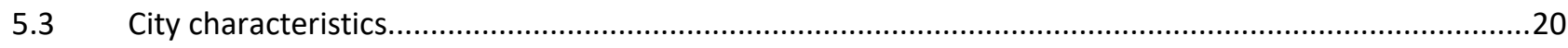

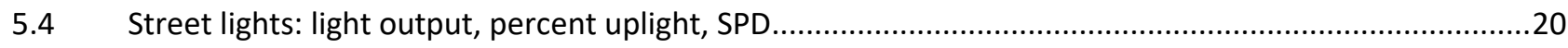

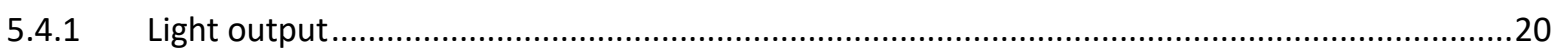

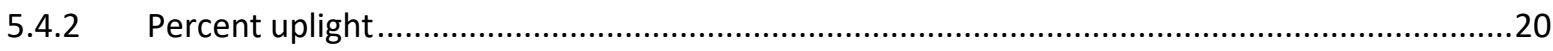

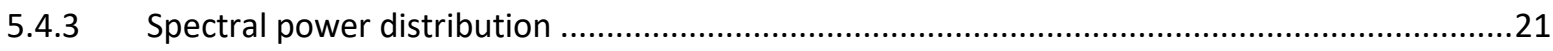

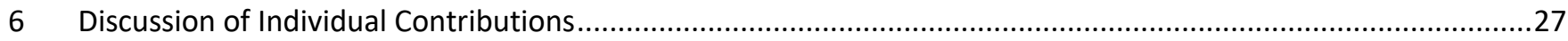

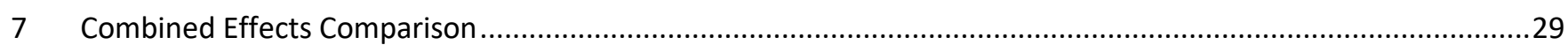

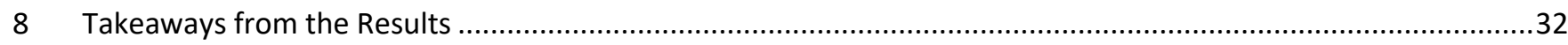

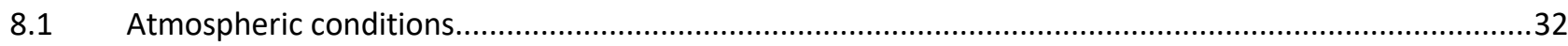

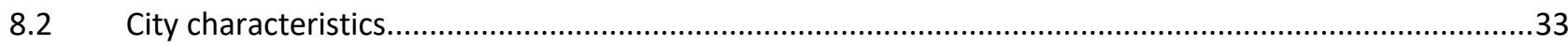

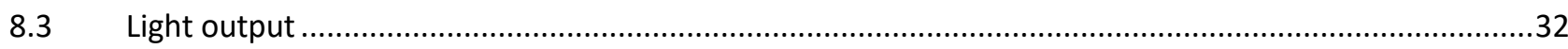

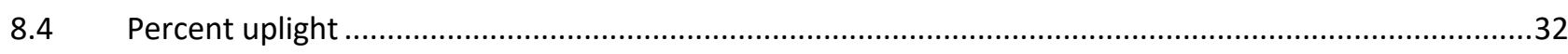

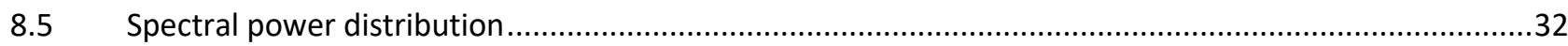

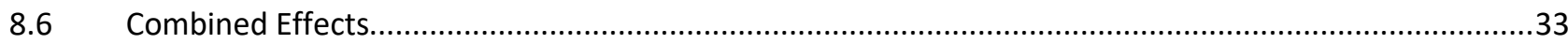

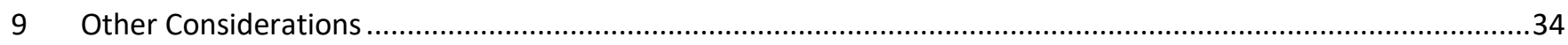

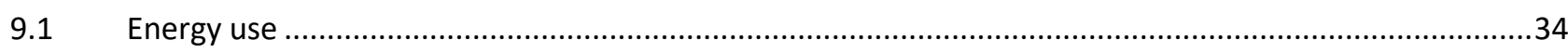

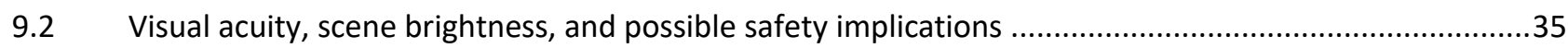

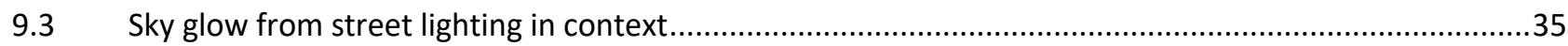

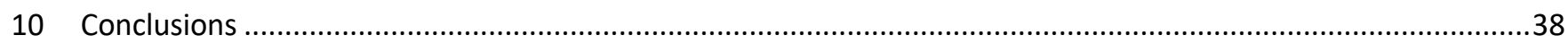

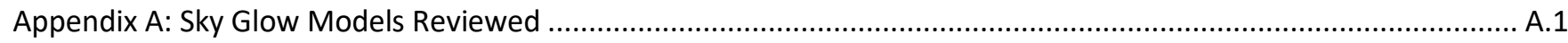

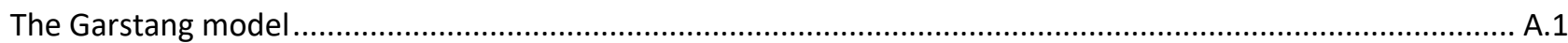

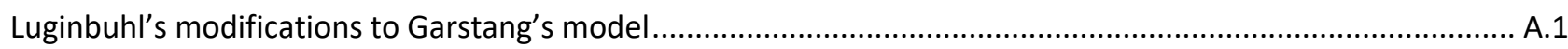

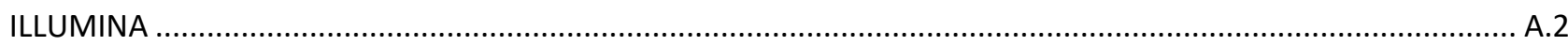

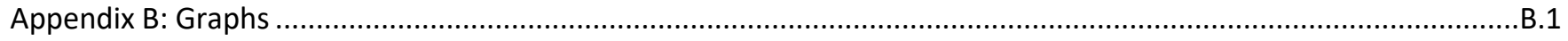




\section{Introduction}

Humanity began illuminating the night with fire-based sources long before the dawn of civilization. This practice continued, albeit with different fuels and increasingly sophisticated technology, through the millennia until the turn of the $20^{\text {th }}$ century, when electric lighting sources became commercially viable. Since then, electric lighting has dominated the nighttime illumination of both interior and exterior spaces. This development founded a structural component of civilization that continues to contribute much to our modern lives, but it has also altered many characteristics of the night environment, and brought with it less desirable effects.

One of these effects is sky glow, the visible background luminance (brightness) in the atmosphere above towns and cities that obscures the view of stars from the ground. ${ }^{1}$ Many lighting sources contribute to sky glow, from both interior and exterior locations, to the degree that each individual installation allows it. Some amount of sky glow is unavoidable if our nighttime environments are to be illuminated; however, the levels over many modern urban areas (that generally also spill over into neighboring non-urban areas) are not an inherent requirement of nighttime illumination, but often rather a result of insufficient attention to proper design and control of the light needed for a specific application. The underlying reasons do not necessarily reflect carelessness, but may instead derive from decisions made to control costs.

The first several decades of electrically illuminating the night primarily saw the use of broad-spectrum, or "white" light sources, in both interior and exterior applications. Beginning around the 1970s, more narrowspectrum products like high-pressure sodium (HPS) and low-pressure sodium (LPS) appeared on the market and started to claim market share due to their higher efficiency and improved lumen maintenance relative to the earlier mercury vapor and incandescent sources in common use at the time. In the 1990s, metal halide lamps, which exhibited a better color-rendering white light, were used in some prominent pedestrian areas, although its application was limited by the lamp's shorter life and lower efficacy compared to HPS. The mid-2000s saw the beginnings of a wider interest in more broad-spectrum sources with the advent of solid-state lighting (SSL) or light-emitting diode (LED) technology. LEDs are favored over the incumbent technologies due not only to their significantly reduced energy use and improved lumen maintenance, but also for their improved color rendition properties and high precision of optical control, among other benefits. It is also useful to note that even at the time LEDs became commercial, many street and roadway lighting systems continued to employ mercury vapor lights and even some incandescent sources in older installations. Thus, some sites have been using essentially the same technology for more than 100 years.

Broad-spectrum light sources emit more radiant energy in the shorter wavelengths of the visible spectrum, which may include varying components of violet, blue, cyan, and other colors, compared to narrower-spectrum sources that emit much more of their output in longer wavelengths like red and yellow. Because shorter wavelengths scatter more readily in the Earth's atmosphere than longer wavelengths, along with certain biological sensitivities to shorter wavelengths, concerns have been raised regarding the potential impact from converting exterior lighting sources with low, short wavelength content (primarily HPS) to broad-spectrum LED.

1 This study calculates sky glow impacts in terms of diffuse irradiance $\left(\mathrm{W} / \mathrm{m}^{2}\right)$ or illuminance (scotopic-cd/ $\mathrm{m}^{2} / \mathrm{sr}$ or scotopic-lux) on the horizontal plane resulting from any incremental brightness of the sky from the natural background, collected from all angles and directions. Radiance is directional and so may vary depending on the observer's direction of view. 
Street lighting is a common focus of these concerns, which include a possible increased loss of night-sky visibility from sky glow and potential health issues from exposure to the associated higher levels of light.

While the science behind short wavelength light and its impacts evolves, significant uncertainty remains regarding how the ongoing transition to LED luminaires will contribute to these situations. Many current impacts have been projected in the literature, but take a highly simplified approach, often directly substituting an assumed spectral power distribution (SPD) of an LED source for that of an HPS source, while holding all other factors (e.g., light output and distribution) constant. Such simplification directly conflicts with actual field experience in the U.S., which reveals that such factors usually change significantly during a lighting conversion.

The U.S. Department of Energy Solid-State Lighting Program has therefore undertaken a review of sky glow models over the last 18 months and consulted with several specialists in atmospheric scatter and sky visibility to identify the most critical factors that influence sky glow. Atmospheric models are complex and often require advanced computers to adequately estimate the effects of different factors on sky glow, particularly as the number of factors considered increases. In this study, the factors considered as contributors to the presence of sky glow include some that are inherent to the street lighting system and others external to it, as given below.

\section{Street Light Factors:}

- Luminaire light output

- Luminaire light distribution, including amount of uplight

- Spectral content of the emitted light

\section{External Factors:}

- Atmospheric conditions (clear, cloudy, and a range of humidity, turbidity, and particulate content)

- City size and lighting density

- Observer location relative to the city center

- Certain fixed assumptions, such as reflectance values of the ground surface onto which the light is projected

Other factors not considered here due to the associated computational burden, but that would be relevant for an actual location, include the following:

- Shape of the city and distribution of light sources and their associated intensities

- Variations in atmospheric particulate size, shape, and distribution

- Multiple scattering of wavelengths (only single scattering was taken into account in this study)

- Light extinction (absorption or blocking) effects of buildings, trees, and terrain

This study estimates the typical changes to sky glow in the U.S. occurring as a result of converting HPS street lighting to broader spectrum sources, with particular focus on LEDs, and reports the contributions relative to HPS baseline conditions. It is important to note that the relative comparisons of sky glow impact apply only to the street lighting system's contribution to the sky glow over a city, and not to the impact that all city light sources have on that sky glow. Section 9.3 discusses this in more detail. Companion issues regarding the energy and lighting quality tradeoffs typically associated with various means of reducing sky glow are also presented. However, associated biological impacts of the short wavelengths are outside the scope of this study. 


\section{A Brief Background on Sky Glow}

A large body of research exists on the sources, severity, and consequences of sky glow. ${ }^{2}$ Many of these studies describe modeling methodologies that incorporate estimates of different light reflectances and scattering properties, or procedures for measuring sky glow to estimate impacts to night sky visibility. A brief discussion of concepts and terms commonly used is helpful for understanding the relevant issues.

\subsection{Astronomical observation}

Stellar brightness is described in units of visual magnitude, an inverse logarithmic scale where smaller numbers are assigned to brighter objects. The scale was first developed by ancient Greek astronomers who subjectively divided stars into six categories of visibility, the brightest assigned a magnitude of 1 and the faintest a value of 6 . Fast-forward a couple of millennia and much of our current quantification scheme is still based on this rudimentary method, although much more sophisticated techniques of measurement and calculation have been developed in the time since, including expansion of the scale beyond 1-6.

To be visible, stars and other celestial bodies must offer sufficient contrast from their background. This means that as background luminance or sky brightness increases, an increasing range of objects become obscured from view. On a clear, moonless night in an environment free of light pollution, the stars just at the edge of unaided visibility to a completely dark-adapted human eye can fall between magnitudes 6 and 8 . With a telescope, this range can extend to magnitude 20 (positive) and beyond. To illustrate the other end of the scale, our sun is at magnitude -26.7 (negative).

Sky brightness is often described in terms of the limiting magnitude, the threshold at which objects with lower brightness (i.e., larger positive magnitude) can no longer be distinguished. The number of visible objects at a given brightness is inversely related to that brightness, so that any increase in limiting magnitude compared to a pristine environment free of background light quickly eliminates many lower-brightness celestial objects from view.

\subsection{Reported units of measurement}

Units of measurement produced by the various methods and devices used to estimate and report sky glow require conversion depending on their intended use. Figure 1 shows a nomogram available from the Dark Skies Awareness ${ }^{3}$ website that ties several commonly used metrics together and enables a helpful first-order conversion among them. ${ }^{4}$

2 For example, see the list of references provided by Christopher Kyba, which date as far back as 1970 (note this list is no longer maintained): http://userpage.fu-berlin.de/ kyba/literature/sky_glow.html.

Dark Skies Awareness: http://www.darkskiesawareness.org/.

4 This study uses different measures of sky brightness (irradiance in $\mathrm{W} / \mathrm{m}^{2}$ and scotopic illuminance in scotopic-cd/m $/ \mathrm{m}^{2} / \mathrm{sr}$ ) reaching an observer's point of view from the increased sky brightness in all directions, which do not appear in the nomogram. 


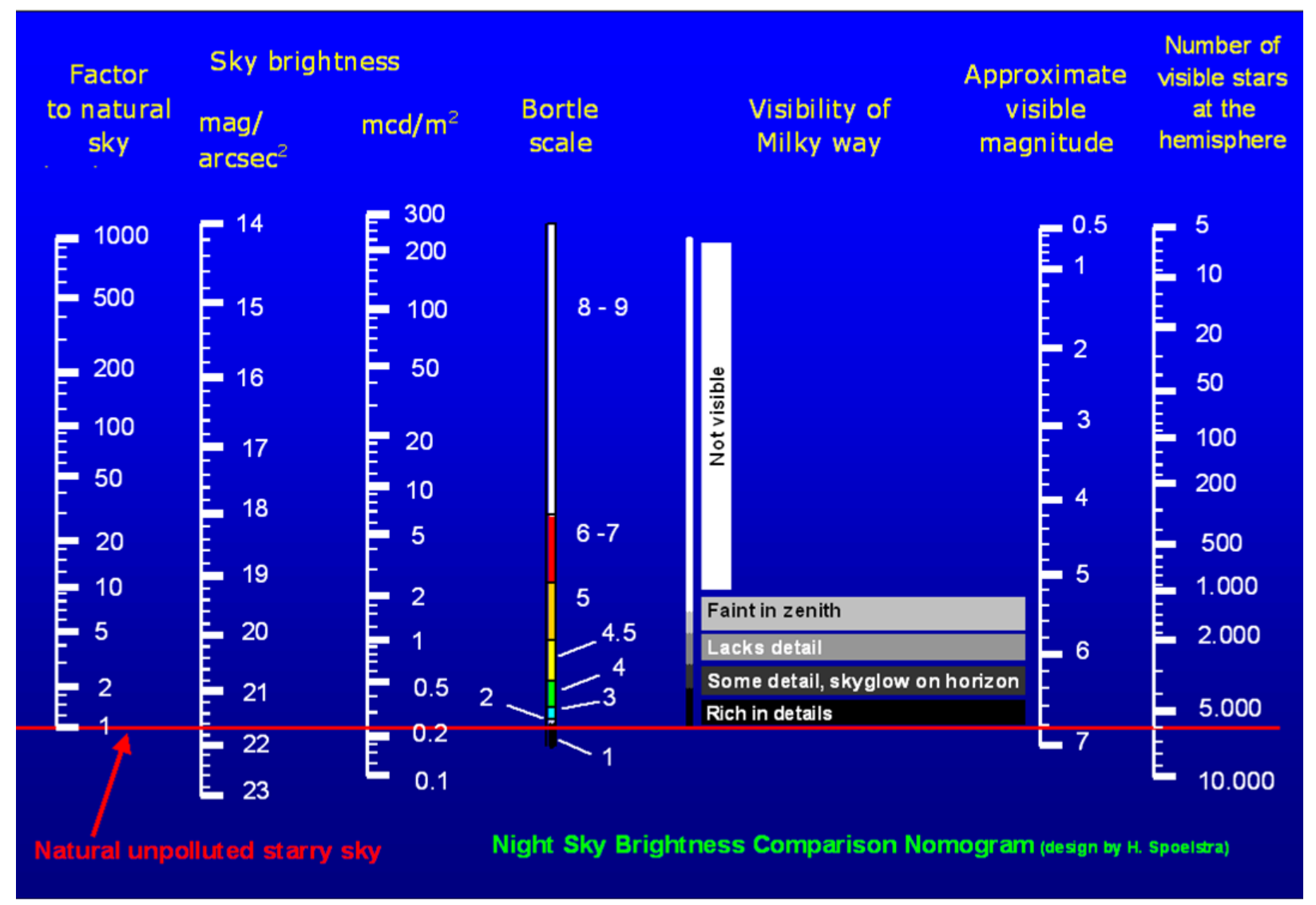

Figure 1 Night sky brightness comparison nomogram. (Source: http://www.darkskiesawareness.org/nomogram.php.)

\subsection{Atmospheric scattering}

Sky brightness is increased by both natural (e.g., the Milky Way, the moon, space dust) and anthropogenic light sources, as well as by direct radiation and reflection/scattering from air molecules and airborne aerosols within the atmosphere. Rayleigh scattering occurs when visible light wavelengths encounter particles of a much smaller relative size (e.g., atoms and molecules). Such particles act as single dipoles, given the intensity of scattered radiation is identical in opposite (i.e., forward and backward) directions. The light is scattered diffusely according to a mathematical relationship between the particle size and wavelength, making Rayleigh scattering wavelength-dependent. The nitrogen and oxygen molecules that make up the vast bulk of our atmosphere are of a size that is most relevant to the blue end of the visible spectrum, and thus are responsible for the blue appearance of our sky. ${ }^{5}$ This same relationship applies to light produced by electric sources.

In contrast, Mie scattering occurs when the particle size is similar to or larger than the wavelength, as applies to aerosols (e.g., water droplets, dust, smog, salt). As atmospheric aerosol content increases, the amount of scattering also increases but the wavelength dependence of the resulting sky glow decreases. Mie scattering occurs more uniformly across the visible spectrum (giving clouds their characteristic white color) and has a significant forward bias. Backscatter is even more suppressed if particles become irregularly shaped. Mie

\footnotetext{
Rayleigh scattering for wavelengths at $400 \mathrm{~nm}$ is 9.4 times as great as that at $700 \mathrm{~nm}$ for equal incident intensity. See: http://hyperphysics.phy-astr.gsu.edu/hbase/atmos/blusky.html. Furthermore, our perception of the sky as "blue" is more a result of the sensitivity of the human retina than the sky's actual wavelength content. Were our eyes sufficiently sensitive to the corresponding wavelengths, the sky would instead appear violet. See: http://www.spc.noaa.gov/publications/corfidi/sunset/.
} 
scattering's contribution to sky glow is greatest for light projected at angles near the horizon, where small particle forward-scattering directs light back to earth. ${ }^{6}$

Electric light finds its way into the atmosphere via direct emission, scattering, and reflection. Many modern outdoor luminaires now restrict all light emission to below the horizontal plane (through the luminaire design), so that the primary upward component of light from the luminaire results from ground reflection. Reflectance values vary by type of ground cover, but commonly used average values range between $12 \%$ and $15 \%$. Outdoor luminaires that most commonly cause direct emission to the sky are as follows:

- Traditional drop-lens fixtures designed to distribute light at high angles for extended reach (many, or most of which, are being slowly phased out by state or local ordinances and otherwise updated lighting practices)

- Fixtures pointed into the sky (e.g., some architectural lighting, advertising signage, and angled fixtures like spotlights and sports lighting)

- Decorative fixtures like acorn- or globe-style post-top units, often found in historic districts (see Figure 2)

High-angle downward light (i.e., light emitted within a few degrees below the horizontal plane) that reaches the atmosphere does so primarily through scattering on its extended path toward the ground, but a commonly held position is that this light is typically extinguished by buildings and vegetation before its contribution becomes significant. ${ }^{9}$ Some sky glow models therefore ignore the contribution of high-angle downward light entirely, while others similarly discount upward light emitted near the horizontal plane.

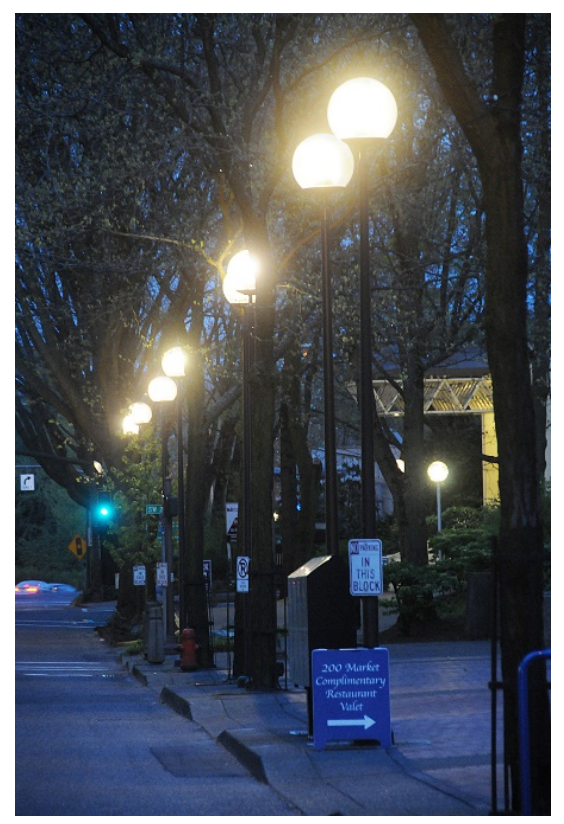

Figure 2 Globe fixtures.

See: http://hyperphysics.phy-astr.gsu.edu/hbase/atmos/blusky.html for illustrations of the effects of particle size and incident angles. R. Stemprok. 'Real World Background Luminance for Objects to be Viewed by Night Drivers.' Electric Power Research Institute (EPRI) RFR 053926, 2004.

E. Dobos. 'Albedo.' Encyclopedia of Soil Science. DOI: 10.1081/E-ESS 120014334, 2003.

C. Luginbuhl et al. 'From the Ground Up II: Sky Glow and Near-Ground Artificial Light Propagation in Flagstaff, Arizona.' Publications of the Astronomical Society of the Pacific, vol. 121, 2009, pp. 204-212. 


\section{Luminaire Characteristics Contributing to Sky Glow}

Three main characteristics of the luminaire determine its contribution to sky glow. Each of these elements can be adjusted through their initial specification, and sometimes dynamically throughout their operation, to help control their impact.

\subsection{Light distribution}

Luminaires are traditionally designed with reflectors and optics to create a particular light distribution that fits the needs of a given application. The resulting distribution typically alters the path of much of the original light output from the source (lamp or LED). Lamp-type sources are often referred to as omni-directional emitters because the lamp yields light output in nearly all directions. Without control of this output using reflectors and lenses, much of the output would be wasted on unintended targets or lost to the night sky, and moreover would likely cause glare for an observer (akin to illuminating a room using a bare bulb).

In contrast, most state-of-the-art LED products emit light out of a single side of the chip to form a directional emission pattern. This light usually still requires some redirection to shape the distribution to the application, but in general the need for redirection is much lower. A distinct advantage of this characteristic is that generally more of the light output goes where it is wanted and less where it is not wanted. Decreasing stray light not only reduces the incidence of light trespass that traditional sources sometimes cause, but also contributes less light to the night sky.

A related improvement of most modern street lighting fixtures (applicable to both LED and conventional sources) is the ability to eliminate virtually all output above the horizontal plane. Older fixtures frequently used drop-lenses to extend the reach of their illumination between mounting poles or into other distant areas, but modern flat lens optics have greatly reduced the need for this approach (Figure 3).

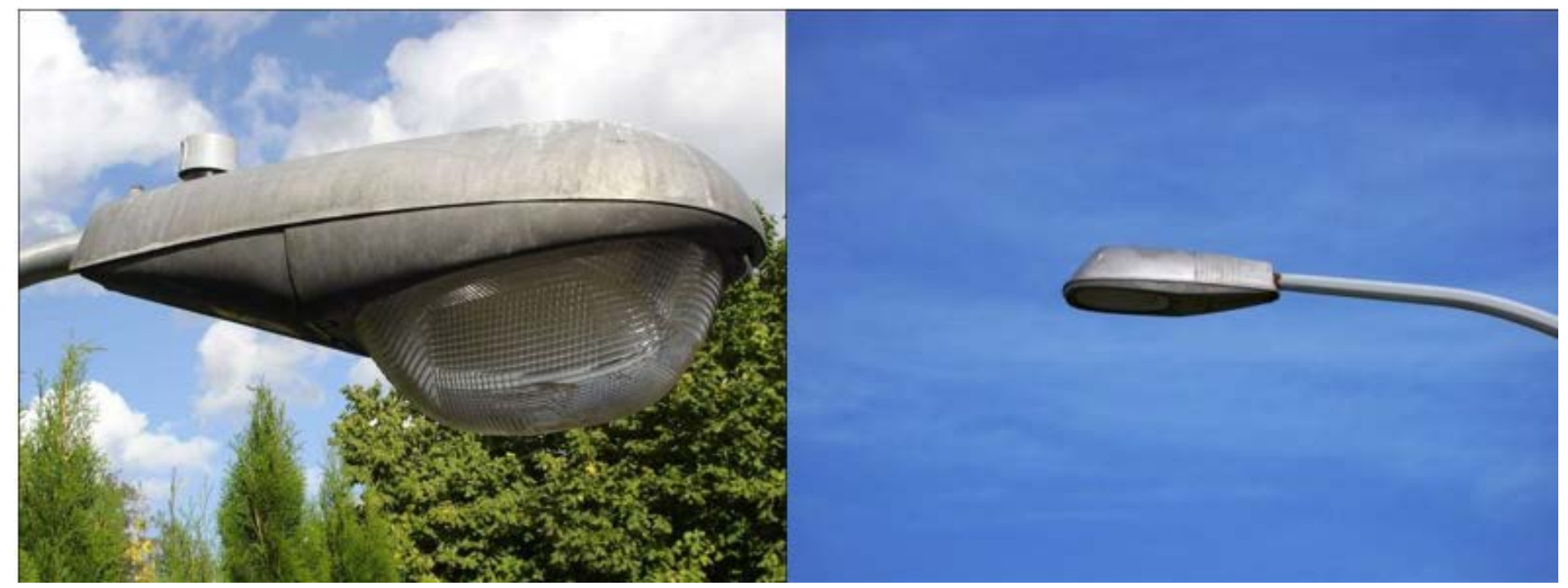

Figure 3 Drop-lens (left) and flat-lens (right) cobra head street light fixtures. 
Drop-lens fixtures emit a highly visible portion of their output directly into the sky, as is evident by direct views of the light source from above. In contrast, the light sources in flat-lens fixtures are not usually visible from above (Figure 4).

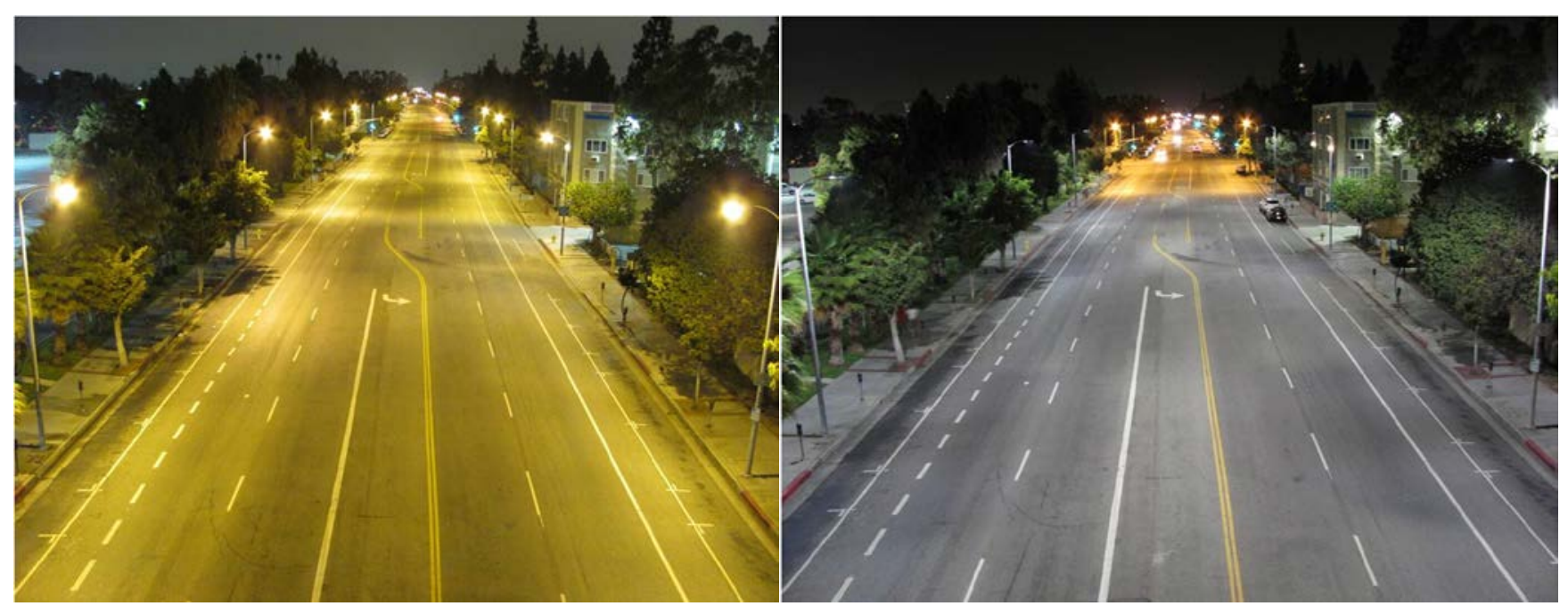

Figure 4 Absence of uplight from modern luminaires at right compared to older drop-lens fixtures at left. (Photo credit: LABSL)

\subsection{Luminaire light output}

Improvements in uniformity that accompany improved distribution also mean fewer shadows in some areas and lower hot spots from excess illumination in others. Together, these benefits enable LED products to generally meet a given lighting application with far fewer lumens than required by traditional lamp-based products. Light output of LED products is often half, or even less, of the light output of incumbent products they are replacing.

\subsection{Spectral content}

A significant body of literature is evolving on the impacts of light at night with respect to spectral content (as reflected in its SPD). As noted, shorter wavelengths tend to scatter more readily in the atmosphere than longer wavelengths, so the spectral content of a light source directly influences sky glow.

The SPD of a source describes its spectral content in terms of radiant power emitted in each wavelength bin across the spectrum. Although SPDs for a given source technology often share similar characteristics, different products frequently have significant variations in output among wavelength bins and furthermore may incorporate additional chips with different properties to enhance certain colors or other qualities (Figure 5). Most source technologies are therefore inaccurately represented by an assumed set of monolithic properties, especially when products using that technology are available in widely different SPDs, often even at the same CCT. Despite the common use of

Figure 5 PC-amber-cyan-violet flat lens chip array (ledengin.com). CCT as an indicator of SPD, the specific spectra contained in the sources are more directly relevant to sky glow than the light's apparent hue. 


\section{$4 \quad$ Selected Sky Glow Model: SkyGlow Simulator}

\subsection{Theory}

Miroslav Kocifaj developed the model SkyGlow Simulator in 2007. ${ }^{10,11}$ (An example of the graphical user interface is shown in Figure 6 and the geometrical setup of the model is shown in Figure 7.) The model is scalable and designed for realistically shaped emitters (e.g., cities or defined land surface areas) as an improvement over point-sources. SkyGlow Simulator models light emitted by ground sources based on the angular distribution of light (e.g., power per unit solid angle per unit projected source area). The surface area (shape and size) of a city is defined through coordinate vertices (latitude, longitude). To characterize the emitted light, the number of individual light sources is defined, along with their spectral and angular radiative characteristics. The model calculates the total ground emission based on total luminaire input wattage, total light output, or the city population (e.g., lumens per person). Essentially, the model combines ground-based light sources, built of many pixels, with a vertically stratified atmosphere (characterized by specific altitudedependent volume scattering and absorption coefficients). Each pixel is characterized by the position with respect to the observer, the total radiant flux, and the spectral radiance as a function of zenith angle.

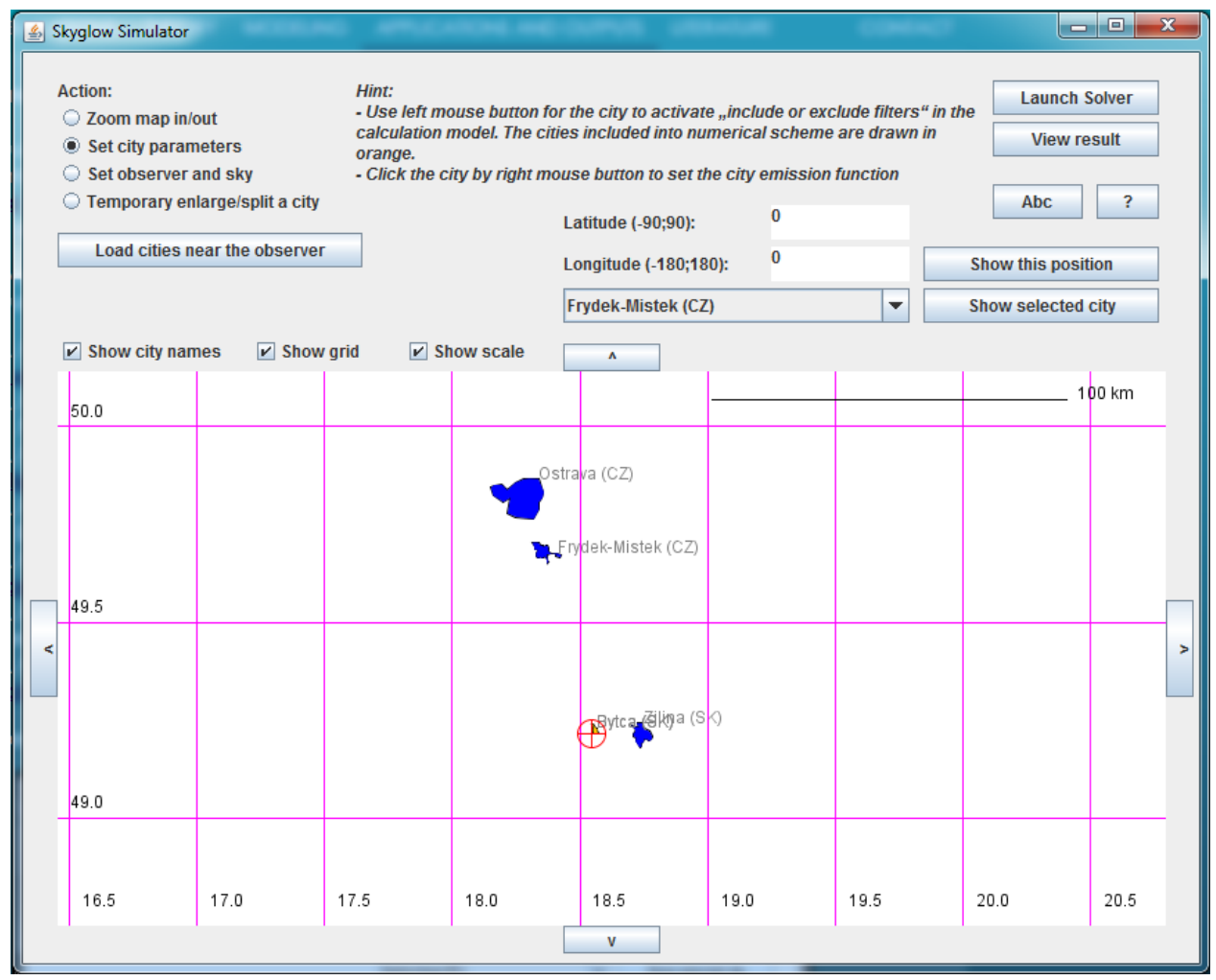

Figure 6 Graphical user interface for the model SkyGlow Simulator.

10 M. Kocifaj. 'Light-pollution model for cloudy and cloudless night skies with ground-based light source.' Applied Optics, vol. 46, no. 15, 2007.

11 SkyGlow Simulator: http://unisky.sav.sk/?lang=en\&page=aplikacia\&subpage=glow. 


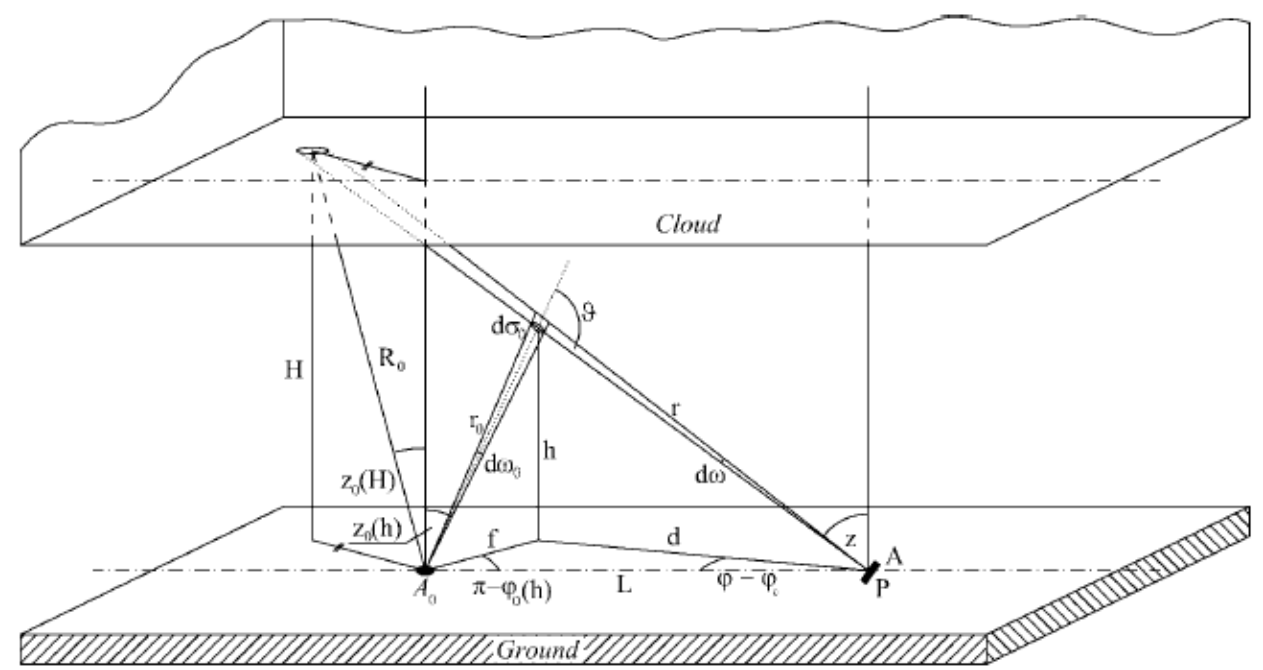

Figure 7 Geometrical setup of the light-pollution model for SkyGlow Simulator.

The light intensity creating the pattern of sky glow in an atmospheric elementary volume is a sum of intensities from all of the beams emitted from different areas under different zenith angles; thus, upward light is an amalgamation of emission distributions. The angular emission function is a key property for evaluating a specific surface area's sky glow; different installations, urban designs, and terrestrial geographies dictate the necessity of multiple functions. Garstang found an approximate formula for the angular behavior of radiation produced by surface light sources, which has become the city emission function most sky glow models use:

$$
B\left(Q, q, z_{0}\right)=2 Q(1-q) \cos z_{0}+0.554 q z_{0}^{4}
$$

The quantity $Q$ is the fraction of light isotropically reflected from the ground, while $q$ is the fraction radiated directly upward, according to the zenith angle $\left(z_{0}\right)$.

Kocifaj and Lamphar point out the lack of validity in using Garstang's original emission function and propose two modified functions. ${ }^{12,13}$ Most critically, Garstang's function overestimates the emissions at low elevation angles because these emissions are efficiently suppressed (by obstacles for beams that propagate along inclined trajectories and by cosine-projection for incident radiation on a horizontally oriented surface). The problem with overestimated emissions to low elevation angles can intensify when solving the radiative transfer equation and when radiance is mistakenly replaced by the radiant intensity function in the boundary conditions. Despite the limitations with Garstang's emission function (GEF), it remains the most frequently used, and, until recently, there was no proposed replacement analytical formula. Given SkyGlow Simulator is based on the Method of Successive Orders of Scattering with finitely-sized light sources, GEF is not as critical and the discussed problems are of less concern. ${ }^{14}$ At the time of this research, the only available city emission functions in SkyGlow Simulator were cosine and Garstang.

12 M. Kocifaj and H. A. Solano Lamphar. 'Angular Emission Function of a City and Skyglow Modeling: A Critical Perspective.' Publications of the Astronomical Society of the Pacific, vol. 128, no. 970, 2016: http://iopscience.iop.org/article/10.1088/15383873/128/970/124001/meta.

13 M. Kocifaj. 'Retrieval of angular emission function from whole-city light sources using night-sky brightness measurements.' Optica, vol. 4, no. 2, 2017, pp. 255-262: https://doi.org/10.1364/OPTICA.4.000255.

14 The Method of Successive Orders of Scattering (MSOS) models the intensity of the scattered signal through a series expansion of many successive orders. While SkyGlow Simulator is based on the MSOS, only the first order, single scattering, is utilized. 
Various atmospheric conditions can be analyzed in the model, including cloudless, overcast, and cloudy skies. The probability that a photon will be scattered into the solid angle of observation is dependent on the optical properties of the atmosphere, characterized by scattering phase functions. Aerosol particles and air molecules are the most important atmospheric constituents responsible for scattering and attenuation of visible radiation. Thus, the overall transmission function reflects the optical properties (absorption, extinction, scattering) of the molecular-aerosol atmosphere. The phase function for molecular scattering is expressed analytically while that for aerosols is obtained numerically; here the molecular scattering is simulated in accordance with Rayleigh theory, and aerosol scattering is approximated by the Henyey-Greenstein function. Aerosols are one of the most unstable atmospheric constituents given that their spatial distribution depends on microphysical properties of aerosols themselves (size/shape distributions, refractive index, and chemical composition). Thus, a set of characteristics is needed (e.g., asymmetry parameter, single scattering albedo, optical thickness of constituents) for the most accurate representation, but most existing models are simplified to an average state of the atmosphere.

The radiative flux due to scattering in a cloud-free atmosphere (or the region underlying the clouds) is calculated separately from the radiant flux directed from a cloud to the observer, based on the spectral reflectance of the cloud. ${ }^{15}$ The total intensity (sum of cloudless and cloudy, as applicable) is calculated per zenith (strong correlation) and azimuth (weak dependence) angle, resulting in an angular distribution of the sky glow that is either unweighted (radiance, $\mathrm{W} / \mathrm{m}^{2} / \mathrm{sr}$ ) or weighted (scotopic luminance, scotopic $\mathrm{cd} / \mathrm{m}^{2}$ ). The angular distribution is represented by a polar plot (Figure 8).

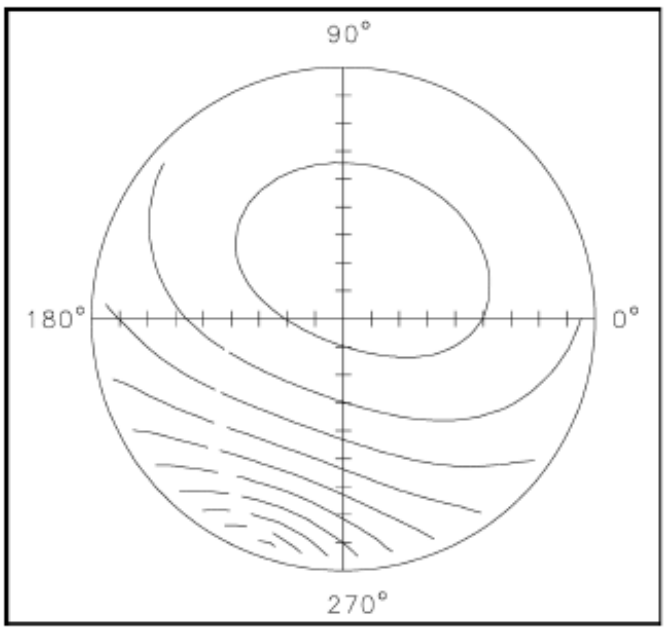

Figure 8 Sky glow polar plot. Isolines are drawn in logarithmic scale to overcome changes to luminance over orders of magnitude. The angle along the circle represents the azimuth of the sky element while the zenith angle is measured from the center to the margin.

Additional outputs include diffuse irradiance $\left(\mathrm{W} / \mathrm{m}^{2}\right.$ ) or scotopic illuminance (scotopic-cd $/ \mathrm{m}^{2} / \mathrm{sr}$ or scotopic-lux) on the horizontal surface and the ratio of zenith radiance or luminance to diffuse irradiance or illuminance (1/sr). The diffuse horizontal irradiance/illuminance value is associated with the overall luminous/radiative flux directed to the observer, independent of the direction at which the light beam is propagated. This value thus does not contain information on dominant light beams. The zenith radiance/luminance relative to the horizontal

15 This theory can only be used for partly cloudy skies as contribution of reflected light depends on shape of cloud; the projected area at a cloud surface changes with the orientation of the normal vector to surface; thus, the numerical implementation must be enhanced to accept geometrical relations. 
irradiance/illuminance, however, is inversely proportional to the steradian and can show how much the sky state changes with distance from a city or town, especially at short distances (e.g., up to a few radii from the city center). This study exclusively analyzed the diffuse irradiance and illuminance values on the horizontal surface. ${ }^{16}$

\subsection{Validation}

Three steps of validation for sky glow models include:

- Comparing a complex model with a simpler but more validated one

- Conducting sensitivity studies to test whether the model predicts a well-known behavior of the phenomenon or to obtain an intuitive understanding of its complex non-linear behavior

- Comparing calculated values with actual measurements

SkyGlow Simulator (formerly denoted $\mathrm{MSNsR}_{\mathrm{Au}}$ ) was compared against ILLUMINA in 2012 and was found to be:

“...well optimized for large-scale simulations. In particular, the grid size is adapted dynamically depending on the distance between a light source and a hypothetical observer. This enables rapid numerical modelling for large territories. $\mathrm{MSNsR}_{\mathrm{Au}}$ is also well suited for the mass modelling of night-sky radiances after ground-based light sources are hypothetically changed. This enables an optimum design of public lighting systems and a time-efficient evaluation of the optical effects related to different lamp spectra or different lamp distributions... Thus a choice of an appropriate model depends mainly on AOD [aerosol optical depth] and CPU requirements." ${ }^{17}$

The second and third steps were addressed through an investigation of the impact of changing spatial and spectral ground-lamp distributions on the hemispherical spectral sky radiance for different observer distances at two observatories: Vartovka and Stará Lesná in Slovakia. ${ }^{18}$ Measurements were taken at Frýdek-Místek in August 2009, which verified and validated the model's performance (Figure 9): "Bear in mind that the focus of the model is not on absolute values in $\mathrm{mcd} / \mathrm{m}^{2}$, but on the (normalized) sky luminance...found that theory fits the measured luminance very well since the mean deviation $\sigma$ is below $2 \% . " 19$

16 M. Kocifaj. 'Retrieval of angular emission function from whole-city light sources using night-sky brightness measurements.' Optica, vol. 4, no. 2, 2017, pp. 255-262: https://doi.org/10.1364/OPTICA.4.000255.

17 M. Aube and M. Kocifaj. 'Using two light-pollution models to investigate artificial sky radiances at Canary Islands observatories.' Monthly Notices of the Royal Astronomical Society, vol. 422, 2012, pp. 819-830.

18 M. Kocifaj, M. Aube, and I. Kohut. 'The effect of spatial and spectral heterogeneity of ground-based light sources on night-sky radiances.' Monthly Notices of the Royal Astronomical Society, vol. 409, 2010, pp. 1203-1212.

19 M. Kocifaj. 'Night sky luminance under clear sky conditions: Theory vs. experiment'. Journal of Quantitative Spectroscopy \& Radiative Transfer, vol. 139, 2014, pp. 43-51. 

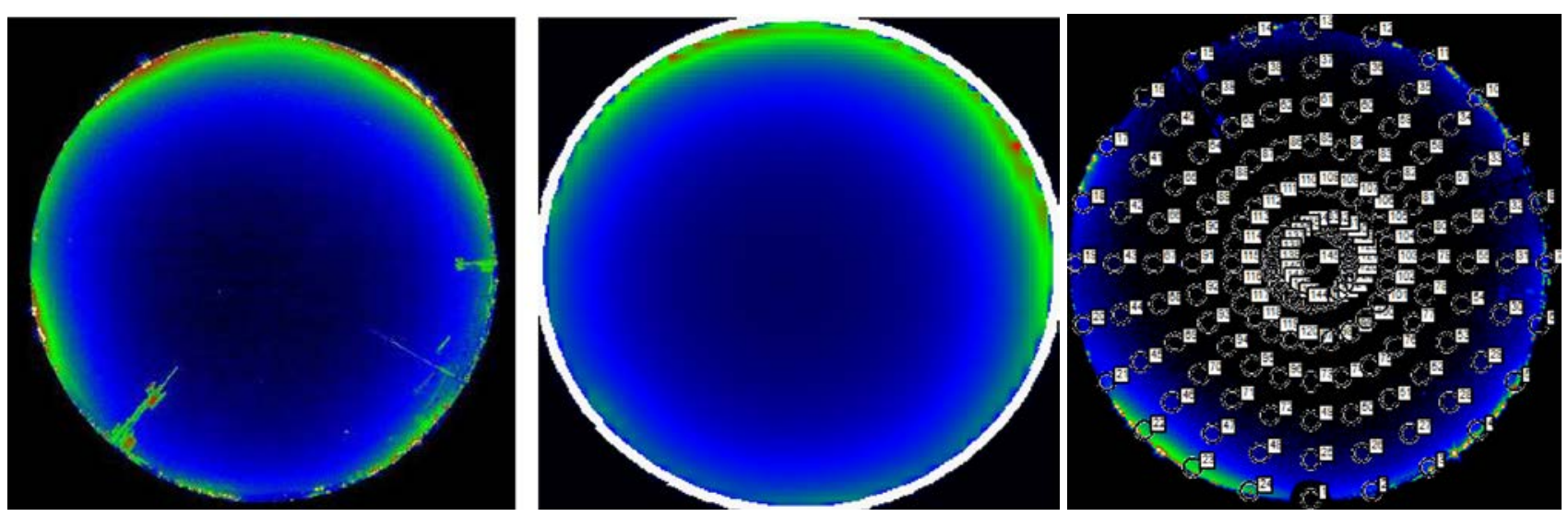

Figure 9 From left to right: measurement of zenith-normalized luminance, reconstructed data of zenith-normalized luminance, and indices and positions of measured sky elements (e.g., where measurements were taken). The luminance values are relative to zenith (assigned a value of 1), with blue indicating 3 times zenith, green 12 times, and red 40 times. 


\subsection{General concepts}

This section discusses general terms and concepts to assist with practical understanding of the modeled results, which follow:

- The tables and figures display the relative impacts of individual variables compared to a stated baseline street lighting system unless otherwise indicated. The ranges (e.g., minimum, maximum) and other statistical values (e.g., $1^{\text {st }}$ quartile, median, $3^{\text {rd }}$ quartile) are calculated between two states of a particular variable (e.g., two uplight percentages), while altering the other factors across their entire ranges. When reviewing relative values, it should be kept in mind that a $10 \%$ reduction in a large number, as often applies to an effect in a nearby observer's location, may exceed a $90 \%$ reduction of a smaller number, such as an effect that might occur at a distance, in terms of absolute impact.

- Absolute sky glow calculations were performed, producing results reported in diffuse horizontal irradiance $\left(\mathrm{W} / \mathrm{m}^{2}\right)$ from the night sky as measured on the ground, in the range of light visible to humans (380 through $780 \mathrm{~nm}$ ). ${ }^{20}$ This is referred to as "unweighted" irradiance because no weighting function such as human visual sensitivity is applied to the calculated values. The results were then also weighted by the human scotopic visibility function, $V^{\prime}(\lambda)$, to yield scotopic illuminance values (scotopic-cd $/ \mathrm{m}^{2} / \mathrm{sr}$ or scotopic-lux) that better represent light visible to the dark-adapted human eye. In each case, the impacts reported in this document take the form of multipliers compared to a baseline value, to indicate the relative impacts.

- The differences between unweighted and scotopically weighted results indicate the potential influence of those results on a) astronomical instrument observations or the response of plants and animals, and b) night sky visibility, respectively. For the light spectra studied in this report, a large difference between the same results when unweighted and weighted indicates a significant presence of short wavelengths around the peak of rod vision (507 nm); little or no difference means a lower presence of such wavelengths.

- Physical parameters often have direct and significant influence; for example, larger cities covering more area tend to produce higher levels of light corresponding to higher total light output, resulting in increased sky glow that extends over longer distances.

- Some other basic physical principles:

o Shorter wavelengths (e.g., violet, blue) scatter more readily than longer wavelengths (e.g., yellow, red) due to the atmosphere's molecular composition ${ }^{21}$; shorter wavelengths thereby have greater influence on sky glow near the source, but rapidly attenuate with distance. Because longer wavelengths are less affected by the molecular elements of the atmosphere, under clear conditions they travel farther and thus have greater influence on sky glow seen by distant observers. ${ }^{22}$

20 Horizontal all-sky irradiance was judged the best metric for purposes of this report, but others like maximum vertical illuminance may also be critical for select sites like environmental preserves. See, for example: D. Duriscoe. 'Photometric indicators of visual night sky quality derived from all-sky brightness maps.' Journal of Quantitative Spectroscopy \& Radiative Transfer, vol. 181, 2016, pp. 33-45.

See, for example: http://www.spc.noaa.gov/publications/corfidi/sunset/.

22 M. Kocifaj. 'A numerical experiment on light pollution from distant sources.' Monthly Notices of the Royal Astronomical Society, vol. 415, 2011, pp. 3609-3615. 
o Water droplets and aerosols like dust, soot, and salt have different effects on the sky radiance because of their much larger sizes and irregular shapes compared to oxygen and nitrogen molecules, the primary scattering agents in a clear sky. ${ }^{23,24}$ Different compounds may also possess different absorption/reflection properties. Higher aerosol loading more uniformly reduces the propagation of all wavelengths, compared to clear conditions.

- Light reaches the sky from a combination of light emitted directly upward (above the $90^{\circ}$ horizontal plane) from the luminaire and light emitted downward (below $90^{\circ}$ ), some of which is then scattered upward or reflected from the ground or other objects (e.g., buildings).

- Some globe and other decorative fixtures emit light straight up (i.e., at zenith), but for most standard cobra head products representing the bulk of installed street lighting fixtures, "uplight" primarily refers to light emitted at a relatively small $\left(0^{\circ}\right.$ to $\left.10^{\circ}\right)$ angle above a horizontal plane drawn level with the aperture of the light source. Light emitted from a drop-lens cobra head street light at higher angles tends to be at much lower intensity than that emitted near horizontal.

- Light emitted or reflected toward zenith travels the shortest path through the atmosphere (the average scale height up to which the uniform molecular atmosphere extends is about $8 \mathrm{~km}$ ) before exiting to space, thus encountering fewer scattering agents than light traveling at lower angles with correspondingly longer atmospheric paths. ${ }^{25}$ Light traveling toward zenith thereby tends to produce less sky glow (under non-cloudy conditions) than light at lower angles.

- Clouds often amplify sky glow for observers within the defined city area, as determined by a complex function of cloud optics and geometry, as well as the position of an individual observer relative to the cloud array.

- Light traveling through the atmosphere is ultimately either absorbed by aerosols, particulates, buildings, or terrain, or exits to space. The scattering effects as the light follows its path are a function of the elements investigated in this study as well as others not covered, such as the complex variations in particulate size and shape listed earlier.

The atmosphere represents a virtually infinite combination of elements when considering the movement of light, particles, or fluids it contains; characteristic inaccuracies are widely recognized in even such common activities as forecasting the weather. Significant simplifications are required in any related modeling effort, and even then models used for these purposes are necessarily both mathematically complex and data intensive.

As this document is designed more to inform the general lighting community (than, for example, the atmospheric or astronomical modeling communities), the descriptions of the modeling methods and results are more tailored toward lighting professionals. The intent is to facilitate a general understanding of the impact of light in the atmosphere resulting from various street lighting scenarios, and how the different characteristics of that light (luminous flux, SPD, and percent uplight) influence sky glow. ${ }^{26}$ The effects of each variable considered

23 For instance, water droplets have spherical or spheroidal forms, thus showing an enhanced backscatter, while dust-like particles are usually of irregular shapes with weak backscatter and various side-scatter features. Soot particles absorb very efficiently, thus they are less efficient in redirecting scattered light back to the ground. On the other hand, salt particles are composed of non-absorbing (or weakly absorbing) species and thus scatter very efficiently. M. Kocifaj et al. 'The effect of spatial and spectral heterogeneity of ground-based light sources on night-sky radiances.' Monthly Notices of the Royal Astronomical Society, vol. 409, 2010, pp. 1203-1212.

25 L. M. Celnikier. 'Understanding the physics of meteoritic descent.' American Journal of Physics, vol. 63, no. 520, 1995, pp. 524-535.

26 Correlated Color Temperature (CCT) is often used as a proxy for spectral content in discussions about sky glow, but is an imprecise measure. Many different combinations of wavelengths can be used to produce a given color appearance, or CCT. This study relies on actual SPDs in interpreting the calculations because the potential differences among products even at the same CCT may result in wide variations in atmospheric effects. 
in this study are described in general terms below, with emphasis on the conditions where the effects are significant:

- Three hypothetical cities were defined, spanning a small city to a large metropolis, believed to be reasonable representations of typical U.S. cities. The information about each is included in Table 1. All cities are unique in their particular shape and dimensions, introducing significant variation among actual locations. To keep the current effort manageable, however, each hypothetical city was simplified to a uniform, circular shape. There is a noticeable range in lighting density, which reflects differences in city design; City2 has the lowest density and meets the qualification as an International Dark-Sky City.

Table 1 Characteristics of hypothetical cities modeled.

\begin{tabular}{|lrrrr|}
\hline & $\begin{array}{c}\text { Radius } \\
(\mathrm{km})\end{array}$ & $\begin{array}{r}\text { City Area } \\
\left(\mathrm{km}^{2}\right)\end{array}$ & No. Fixtures & $\begin{array}{r}\text { Lighting Density } \\
\left.\text { (fixtures per } \mathrm{km}^{2}\right)\end{array}$ \\
City1 & 1.4 & 7 & 342 & 52 \\
City2 & 7.2 & 164 & 3,500 & 21 \\
City3 & 8.6 & 232 & 39,884 & 172 \\
\hline
\end{tabular}

- Two observer distances were selected, the first at the city perimeter (but still within the city, hereafter referred to as the "near observer") and the second $40 \mathrm{~km}$ from the center of the city (well outside the city, hereafter referred to as the "distant observer"). For consistency, the observer positions were uniformly located due east of the city center, at the same cardinal direction relative to center.

- Garstang's city emission function ${ }^{27}$ was used to model how light propagates into the atmosphere. Among the variables that can be specified in the model, two address the direction of light emitted from light sources: 1 ) the fraction of light emitted downward and isotropically reflected (assuming a uniform $15 \%$ ground reflectance), and 2) the fraction of light radiated directly upward (above $90^{\circ}$ ). Per Garstang's function, if the luminaire emits no light upward, then the only light reaching the sky is that reflected from the ground according to a cosine function (represented by the dashed line in Figure 10). If there is uplight, the direct uplight is emitted according to the function represented by the dotted line; the solid line represents the combined product of the downward-reflected and upward-emitted quantities.

27 R. H. Garstang. 'Model for Artificial Night-Sky Illumination.' Publications of the Astronomical Society of the Pacific, vol. 98, no. 601, 1986, pp. 364-375. 


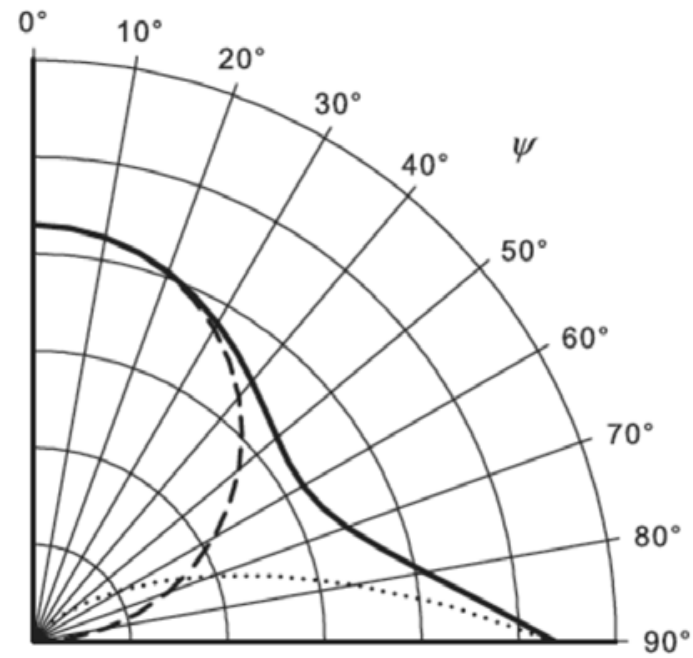

Intensity $(\mathrm{Im} / \mathrm{sr})$

Figure 10 Garstang's city emission function. If the luminaire emits no light upward, then the only light reaching the sky is that reflected from the ground according to a cosine function (represented by the dashed line). If there is uplight, the direct uplight is emitted according to the function represented by the dotted line; the solid line represents the combined product of the downward reflected and upward emitted quantities.

- Light output, SPD, and percent uplight are the variables used to specify the fixture characteristics in the model. The effect of light output was tested using normalized values of 500 and 1,000 Im/fixture. ${ }^{28}$ These values were run and compared for every scenario (e.g., for all source types/technologies). The 11 SPDs listed in Table 2 were selected to represent incumbent technologies and various LEDs. An equal energy SPD (all wavelengths emitted at equal energy) was included to compare associated sky glow effects on a granular wavelength basis, in $5 \mathrm{~nm}$ spectral bands. The scotopic-to-photopic (S/P) ratios relative to HPS for the LEDs ranged from 0.70 to 3.13 , and are listed here because this metric is referenced as a means to evaluate light sources for sky glow potential in some approaches to street lighting design. ${ }^{29}$ Generally, light sources with more emission around $507 \mathrm{~nm}$ (the peak of the human scotopic luminous efficiency function) will have higher S/P ratios. the wide variation of product outputs within even a single installation and the intent of this study to be more generic in its findings. different values for photopic and scotopic lumens. The ratio of these two values is the scotopic-to-photopic (S/P) ratio. 
Table 2 The 11 selected SPDs, normalized to a maximum output of 1, along with their corresponding CCT values and S/P ratios (relative to HPS).

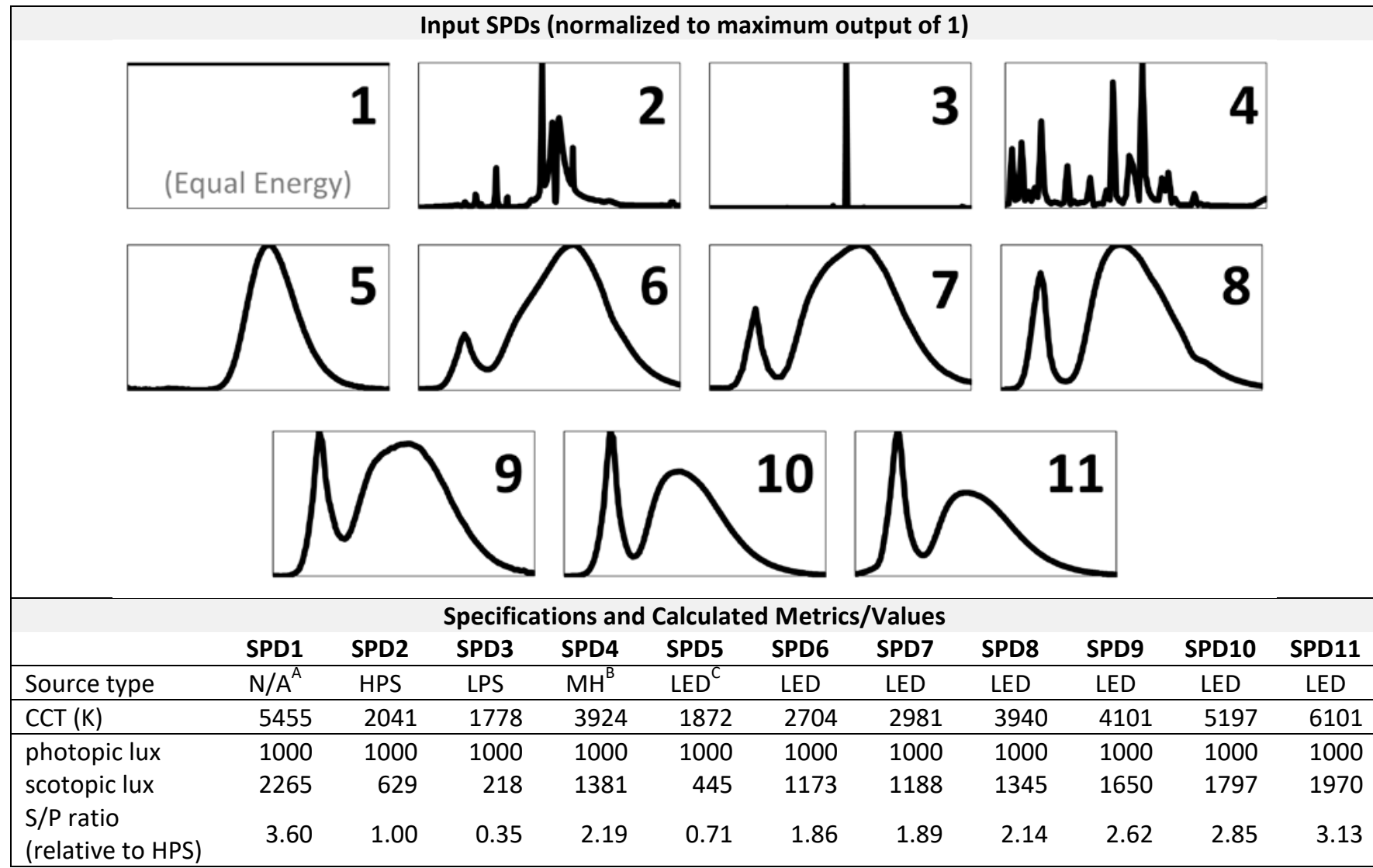

${ }^{A}$ Equal energy

${ }^{B}$ Metal halide

${ }^{\mathrm{C}}$ Phosphor-converted (PC) Amber LED

- Four uplight values were selected for comparison: $0 \%$ uplight roughly represents what were formerly referred to as "full cut-off" fixtures ${ }^{30}$; the values of $2 \%$ and $5 \%$ represent the percent uplight of typical and relatively poor drop-lens cobra head street lights, respectively ${ }^{31}$; and $10 \%$ approximates a good optical quality acorn post-top light. The $10 \%$ value also allows for cross-model comparison since many other sky glow models assume $10 \%$ uplight from street lights. ${ }^{32}$

- The atmospheric conditions modeled include four that are "cloudless" with varying degrees of turbidity, and one of cloud cover (see Table 3). For all conditions, there were no light blocking objects near the horizon. NASA's AERONET station data was used as a reference source for various atmospheric

30 The term has since been deprecated by the Illuminating Engineering Society.

31 A survey conducted by PNNL in February 2017 of 10 HPS drop-lens cobra head street light products from major manufacturers found a range of uplight between $0.1 \%$ and $5.1 \%$, with an average of $2.4 \%$.

32 It is important to note that obstacle blocking was not accounted for in this effort, which may have increased the impact of higher uplight values. Depending on the particular environment, a portion of the uplight (mainly the light travelling emitted near the horizon) could be blocked and absorbed by obstacles, so that eliminating uplight in the luminaires will not have as dramatic of effect. See: M. Aubé. 'Physical Behaviour of Anthropogenic Light Propagation into the Nocturnal Environment.' Philosophical Transactions of the Royal Society - B, vol. 370, no. 1667, 2015. 
conditions in the U.S. ${ }^{33}$ These conditions are loosely based on U.S. cities with the intent to represent a realistic range of conditions.

- The results are reported in both unweighted and scotopically weighted terms to enable their use in analyzing a variety of human, instrument, and non-human biological impacts.

Table 3 Atmospheric conditions modeled.

\begin{tabular}{|c|c|c|c|c|c|c|}
\hline \multicolumn{2}{|c|}{ Atmospheric conditions } & ATM1 & ATM2 & ATM3 & ATM4 & ATM5 \\
\hline \multicolumn{2}{|c|}{ Clouds } & No & No & No & No & Yes \\
\hline \multirow{2}{*}{$\begin{array}{l}\text { Cloud } \\
\text { Details }\end{array}$} & Altitude of the cloud base $(\mathrm{km})$ & $\mathrm{N} / \mathrm{A}$ & $\mathrm{N} / \mathrm{A}$ & $\mathrm{N} / \mathrm{A}$ & $\mathrm{N} / \mathrm{A}$ & 1 \\
\hline & Spectral albedo (select data file) & $\mathrm{N} / \mathrm{A}$ & $\mathrm{N} / \mathrm{A}$ & $\mathrm{N} / \mathrm{A}$ & $\mathrm{N} / \mathrm{A}$ & Altocumulus.cld \\
\hline \multirow{3}{*}{ Aerosols } & Reference aerosol optical thickness ${ }^{\mathrm{A}, \mathrm{B}}$ at $500 \mathrm{~nm}$ & 0.1 & 0.1 & 0.5 & 0.5 & 0.5 \\
\hline & Angstrom exponent ${ }^{A}$ & 0.3 & 1.5 & 0.3 & 1.5 & 1.0 \\
\hline & Scale height for the molecular atmosphere $(\mathrm{km})$ & 8.0 & 8.0 & 8.0 & 8.0 & 8.0 \\
\hline $\begin{array}{l}\text { Data files } \\
\text { for }\end{array}$ & $\begin{array}{l}\text { single scattering albedo } \\
\text { asymmetry parameter }\end{array}$ & \multicolumn{5}{|c|}{$\begin{array}{l}\text { constant_background.ssa } \\
\text { constant_background.ssa }\end{array}$} \\
\hline Horizon & No light blocking objects near horizon & & & & & \\
\hline
\end{tabular}

\subsection{Atmosphere}

Atmospheric conditions play a significant role in both the level and the distribution of sky glow. Clouds have the greatest effect for observers within the city because they act as immediate reflectors, sending reflected light back to the ground. At the same time, these clouds confine light locally, effectively reducing the light observed at some distance from the city. Under cloudy conditions, therefore, distant observers only receive light that is scattered through a long cloud layer and/or directly emitted at angles only slightly above horizontal.

The size, shape, and density of aerosol particles collectively influence the turbidity, which influences the behavior of light traveling through the atmosphere. Although the intensity of scattered light generally increases as atmospheric turbidity increases, that greater aerosol optical depth (AOD) also causes more rapid decay of the intensity of the emitted light beam. Scattering and extinction work in opposite directions, complicating the effect of aerosols on sky glow. Furthermore, optical properties of the aerosol layer depend on particle type and size. Black soot in the air, for example, absorbs much of any incident light and thus creates a darker sky glow, whereas salt aerosols found in coastal regions tend to be diffuse reflectors, making the sky brighter and reducing the contrast of the stars.

Figure 11 illustrates the significant impact to sky glow from dense atmospheres, either highly turbid with many different aerosol particle sizes (ATM3-4) or with complete cloud cover (ATM5). Clearer skies with lighter aerosol dosing (ATM2) display almost none of the same effects, being more closely clustered around the baseline value (ATM1 - assigned the relative value of 1.0 in the charts).

33 Aerosol Robotic Network (AERONET): https://aeronet.gsfc.nasa.gov/. 

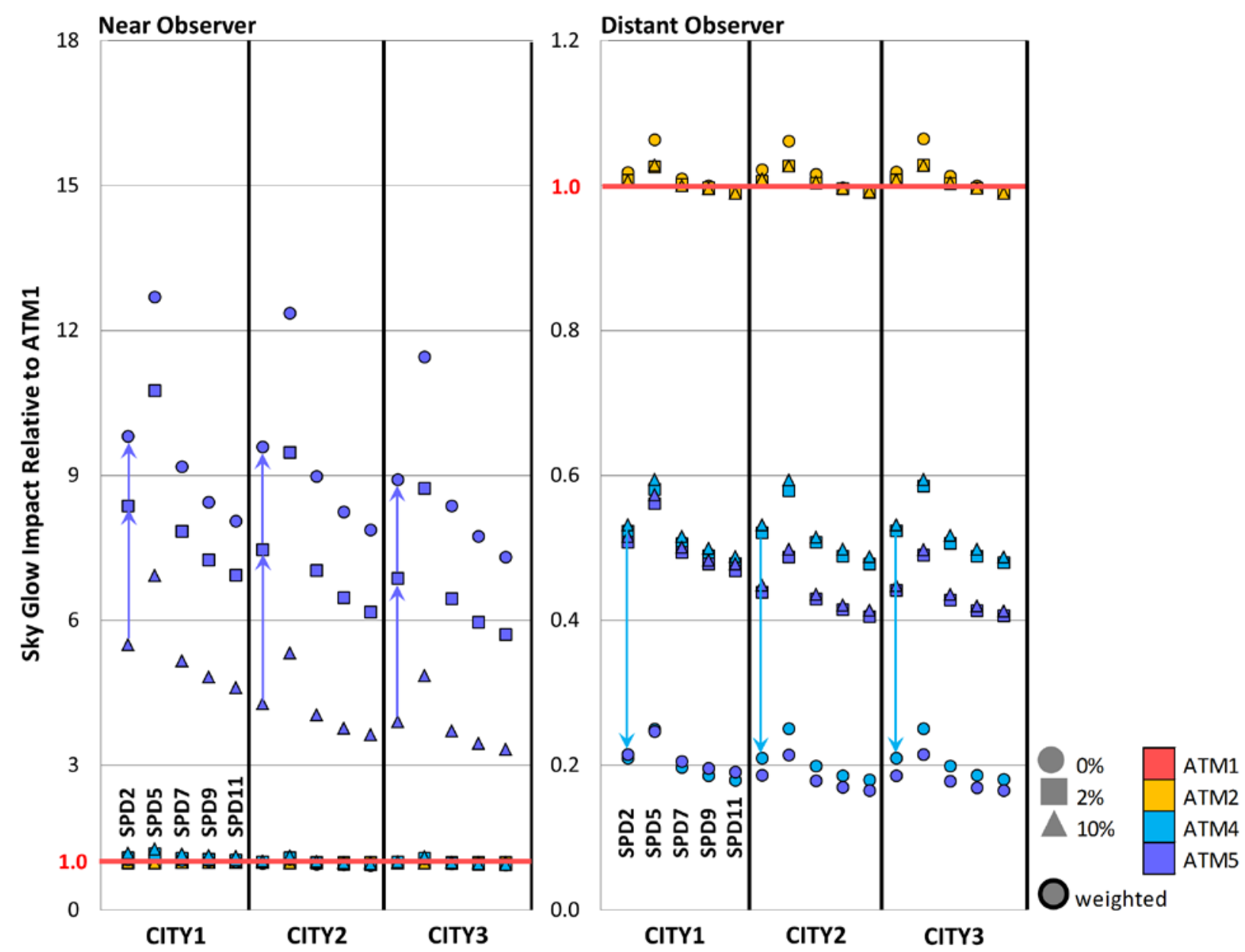

Figure 11 Relative impacts of the atmospheric conditions for the near and distant observer positions. The relative impacts (note the different scales) of select atmospheric conditions (ATM2, ATM4, and ATM5) compared to ATM1 (indicated as red line) are graphed for each city and five select SPDs based on their percent uplight (0\%,2\%, 10\%). Only the scotopically weighted results are graphed. The graph on the left, for the near observer, shows the significant increase in sky glow due to clouds, including the increase in sky glow that comes from decreasing uplight, denoted by the direction of the arrows (for the cloudy condition only). The graph on the right, for the distant observer, shows the influence of more turbulent and cloudy atmospheres in decreasing sky glow. Unlike the graph for the near observer, decreasing the percent uplight decreases sky glow, also denoted by the arrows. (For a complete version of the graph, see Appendix B.)

The atmosphere also affects the impact of uplight from the luminaire. For a near observer under clear conditions, increasing uplight slightly increases sky glow because of the associated increase in scatter, but under cloudy conditions, increased uplight from the luminaire actually reduces the visible sky glow. This contradiction occurs for two reasons: under cloudy conditions, the downward portion of light from the luminaire bounces multiple times between the ground and the overhead clouds, effectively trapping and dissipating it; also, most of the increased uplight is emitted at low elevation angles and undergoes a long trajectory before reaching the cloud base, and thus does not reach the near observer.

For the near observer, the brightness of the night environment noticeably increases under cloudy conditions, increasing even more when luminaires direct all their output downward (i.e., have no uplight). For an observer outside the city, however, the brightness of the night environment decreases as turbidity and clouds increase. Decreasing the uplight reduces sky glow for the distant observer under all atmospheric conditions. 


\subsection{City characteristics}

Three main drivers of sky glow are related to city characteristics: the city area, total light output, and the range of light emission angles that affect other areas within the city. In general, the larger the city, the more complicated the propagation of light and associated scattering in the atmosphere, and the greater the propensity for light from the opposite side of the city (relative to the observer) to propagate at low elevation angles and along longer optical paths. Distant observers receive a collection of light from different angles, depending on their distance relative to the dimensions of the city.

\subsection{Street lights: light output, percent uplight, SPD}

\subsubsection{Light output}

This study compared the effects of light output using two output levels, one twice the value of the other. As the values of all the other input variables were modified in the sky glow calculations, the relationship between light output and sky glow remained linear, such that whenever the street lighting levels were doubled, the resulting sky glow contribution from the street lights also precisely doubled. Conversely, reducing light output reduces the sky glow proportionally.

\subsubsection{Percent uplight}

The percent uplight fraction was varied in the modeling from $0 \%$ to $2 \%, 5 \%$, and $10 \%$. At $0 \%$ uplight, all light in the sky is due to ground reflection and is distributed according to its assumed cosine reflectance function. The other values create a hybrid cosine and low-angle emission pattern (refer to Figure 10).

Figure 12 portrays the modeled impacts of percent uplight. From left to right, each bar shows the minimum, $1^{\text {st }}$ quartile, median, $3^{\text {rd }}$ quartile, and maximum values for all combinations of modeled variables, with the colored areas representing the data between the $1^{\text {st }}$ and $3^{\text {rd }}$ quartiles. These are shown relative to a baseline (represented by the red line at value 1.0) for each comparison of identical conditions, varying only the $2 \%, 5 \%$, or $10 \%$ uplight variable. The charts show that reducing uplight decreases sky glow under all conditions, except for the near observer under cloudy skies. Reducing percent uplight counterintuitively increases sky glow under the cloudy condition when the observer is within the city. (See the discussion on atmosphere in Section 5.2.)

The reduction in sky glow resulting from eliminating uplight is particularly pronounced for the distant observer. Shown at the far left of the bottom charts, sky glow for the distant observer has been reduced by at least $95 \%$ compared to the baseline condition of $2 \%$ uplight. Assuming no obstructions due to buildings, trees, terrain or other factors that change the baseline results for comparison, eliminating uplight has by far the single largest influence of any variable to reduce sky glow for distant observers. 

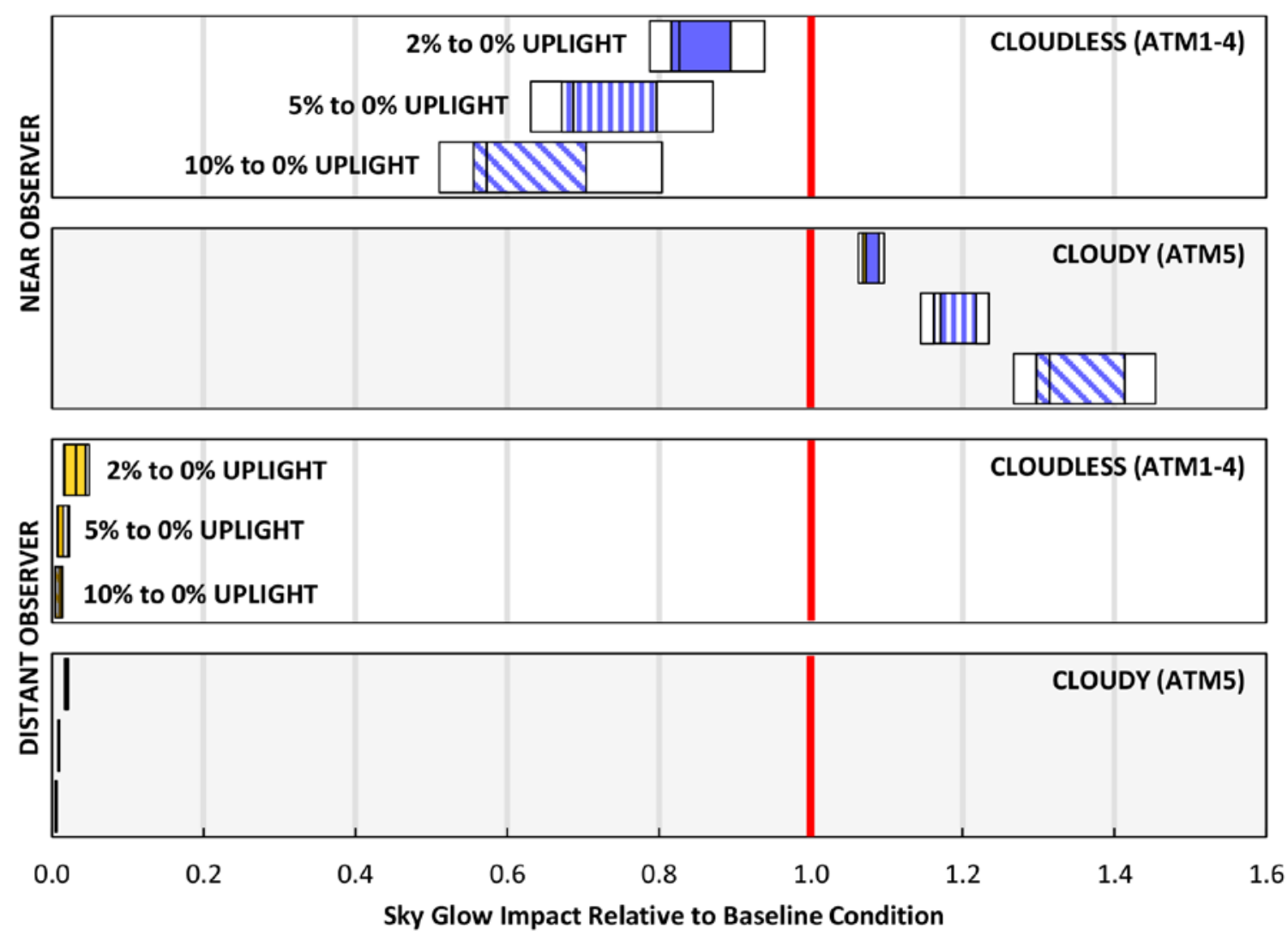

Figure 12 Impact of reducing percent uplight under cloudless sky and cloudy conditions. The top graph is for the cloudless (ATM1-4) conditions while the bottom is for the cloudy (ATM5) condition. The blue bars are for the near observer position while the yellow-orange are for the distant observer position. The solid bars show the impact of changing from $2 \%$ to $0 \%$ uplight, the vertically hatched bars show the impact of changing from $5 \%$ to $0 \%$ uplight, and the diagonally hatched bars show the impact from $10 \%$ to $0 \%$ uplight. Thus, a value of 0.5 indicates that reducing uplight to $0 \%$ from the corresponding baseline results in 0.5 times the sky glow.

\subsubsection{Spectral power distribution}

Table 4 shows the range of impacts of SPD relative to HPS, for all cities and all atmospheric conditions. The table identifies the SPDs by number, with the corresponding CCT values and relative S/P ratios in Table 2. Figure 13 shows the actual SPDs. According to the median values shown in bold, all SPDs increase sky glow compared to HPS except for LPS and PC Amber LED, and scotopic weighting further emphasizes these impacts. These results are due to the increased scatter of the short to very short wavelengths for the unweighted results. The more pronounced impact of short wavelength scatter in the scotopically weighted results is due to the human eye's increased sensitivity to wavelengths near the peak of the scotopic sensitivity curve $(507 \mathrm{~nm})$. Note that the impacts reported are driven by the specific SPD of each respective product. While unweighted sky glow values increase for all light sources with higher contents of very short wavelengths (i.e., violet), the associated influence of very short wavelengths ends when scotopically weighted because the human eye is less sensitive to wavelengths below about $420 \mathrm{~nm}$. 
Table 4 Relative impact of SPD on sky glow compared to HPS for both unweighted and scotopically weighted results, between the two observer positions. HPS (SPD2) is represented by the value of 1 . Cells highlighted in yellow are close to 1 in value; values decreasing from 1 become more greenish while those increasing from one become more reddish.

\begin{tabular}{|c|c|c|c|c|c|c|c|c|c|c|c|c|}
\hline & \multicolumn{6}{|c|}{ Unweighted Results } & \multicolumn{6}{|c|}{ Scotopically-Weighted Results } \\
\hline & \multicolumn{3}{|c|}{ Near Observer } & \multicolumn{3}{|c|}{ Distant Observer } & \multicolumn{3}{|c|}{ Near Observer } & \multicolumn{3}{|c|}{ Distant Observer } \\
\hline & MIN & MEDIAN & MAX & MIN & MEDIAN & MAX & MIN & MEDIAN & MAX & MIN & MEDIAN & MAX \\
\hline SPD1 & 2.0 & 2.5 & 2.9 & 2.0 & 2.2 & 2.4 & 3.4 & 3.9 & 4.2 & 3.2 & 3.6 & 3.9 \\
\hline SPD3 & 0.7 & 0.7 & 0.8 & 0.7 & 0.7 & 0.8 & 0.3 & 0.3 & 0.4 & 0.3 & 0.4 & 0.4 \\
\hline SPD4 & 1.2 & 1.7 & 2.1 & 1.1 & 1.4 & 1.7 & 2.1 & 2.3 & 2.4 & 2.0 & 2.2 & 2.3 \\
\hline SPD5 & 0.9 & 0.9 & 1.0 & 0.9 & 1.0 & 1.1 & 0.5 & 0.6 & 0.7 & 0.6 & 0.6 & 0.7 \\
\hline SPD6 & 1.3 & 1.3 & 1.3 & 1.3 & 1.3 & 1.3 & 1.8 & 1.9 & 1.9 & 1.7 & 1.8 & 1.9 \\
\hline SPD7 & 1.2 & 1.3 & 1.3 & 1.2 & 1.3 & 1.3 & 1.8 & 1.9 & 1.9 & 1.8 & 1.8 & 1.9 \\
\hline SPD8 & 1.0 & 1.2 & 1.3 & 1.0 & 1.1 & 1.2 & 2.0 & 2.2 & 2.2 & 2.0 & 2.1 & 2.1 \\
\hline SPD9 & 1.2 & 1.4 & 1.5 & 1.1 & 1.2 & 1.3 & 2.5 & 2.8 & 2.9 & 2.4 & 2.6 & 2.7 \\
\hline SPD10 & 1.1 & 1.3 & 1.5 & 1.0 & 1.2 & 1.3 & 2.7 & 3.0 & 3.2 & 2.6 & 2.8 & 3.0 \\
\hline SPD11 & 1.2 & 1.6 & 1.8 & 1.1 & 1.3 & 1.5 & 2.9 & 3.4 & 3.6 & 2.8 & 3.1 & 3.3 \\
\hline
\end{tabular}



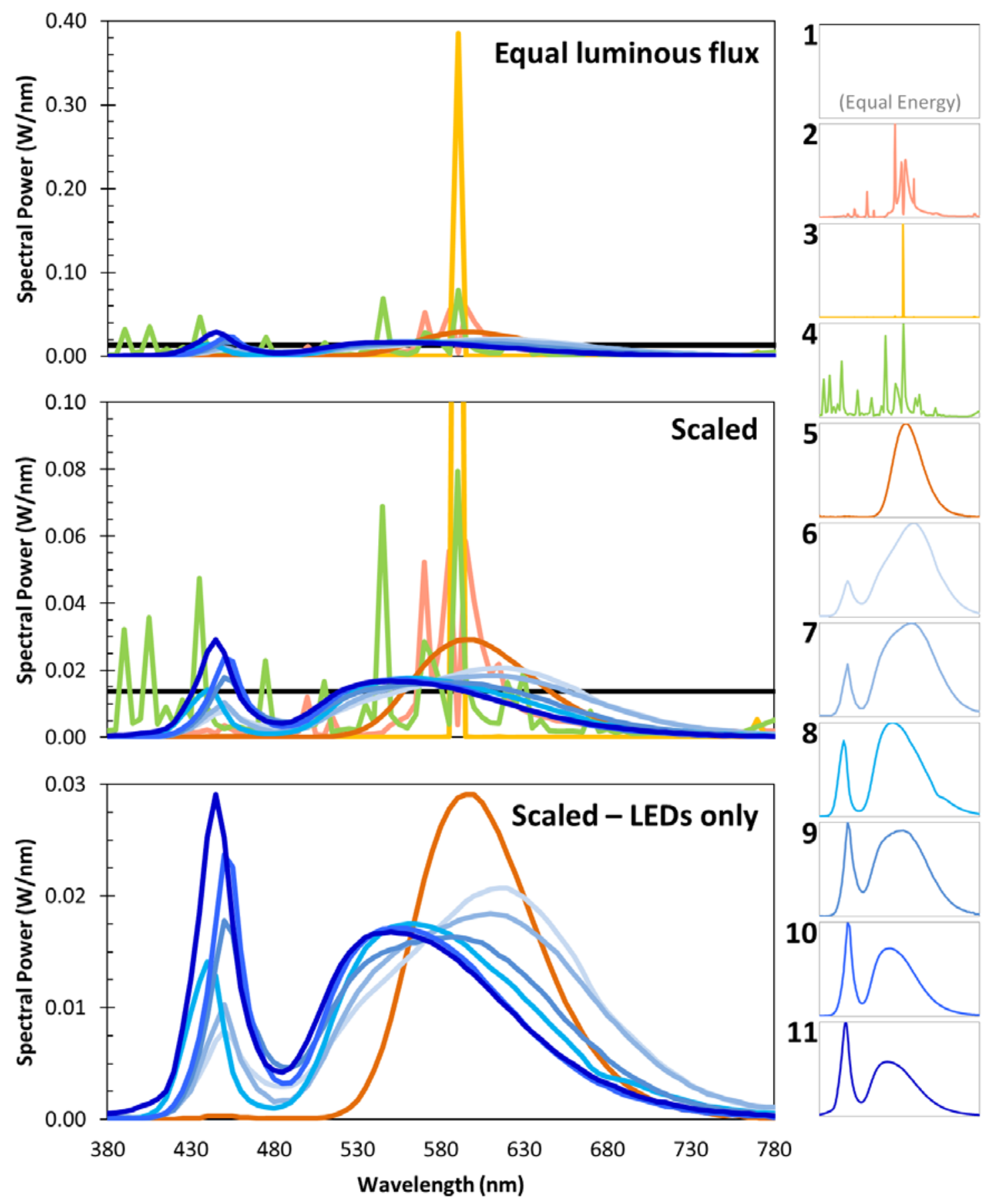

6
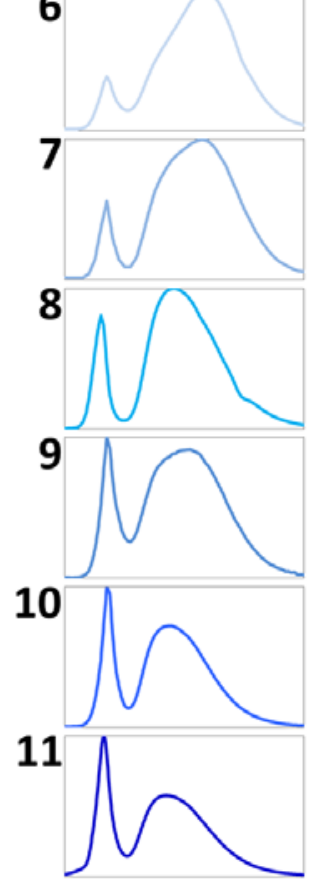

Figure 13 SPDs of light sources modeled in study, graphed from $\mathbf{3 8 0}$ to $\mathbf{7 8 0} \mathbf{~ n m}$ at equal lumen output. The top chart shows the set of SPDs modeled in this study, displaying the overall range in spectral radiant power for all products. The middle chart zooms in to better reveal the variation among the non-LPS waveforms. The bottom chart shows LEDs only. The significant variation among spectral content is evident in all charts. 
Figure 14 shows the same data in the form of bar graphs. The bottom graphs are further divided into atmospheric conditions. Impacts displayed are based on a uniform light output from the source. In these results, city specifications and percent uplight values do not significantly alter the relative impact of each SPD compared to HPS; the impact is more related to the type of result (unweighted versus weighted) and sometimes varies based on observer position (e.g., each SPD has a greater range of impact when the observer is within the city versus outside the city). Scotopic weighting increases the relative sky glow of all light sources except LPS and PC Amber LED.
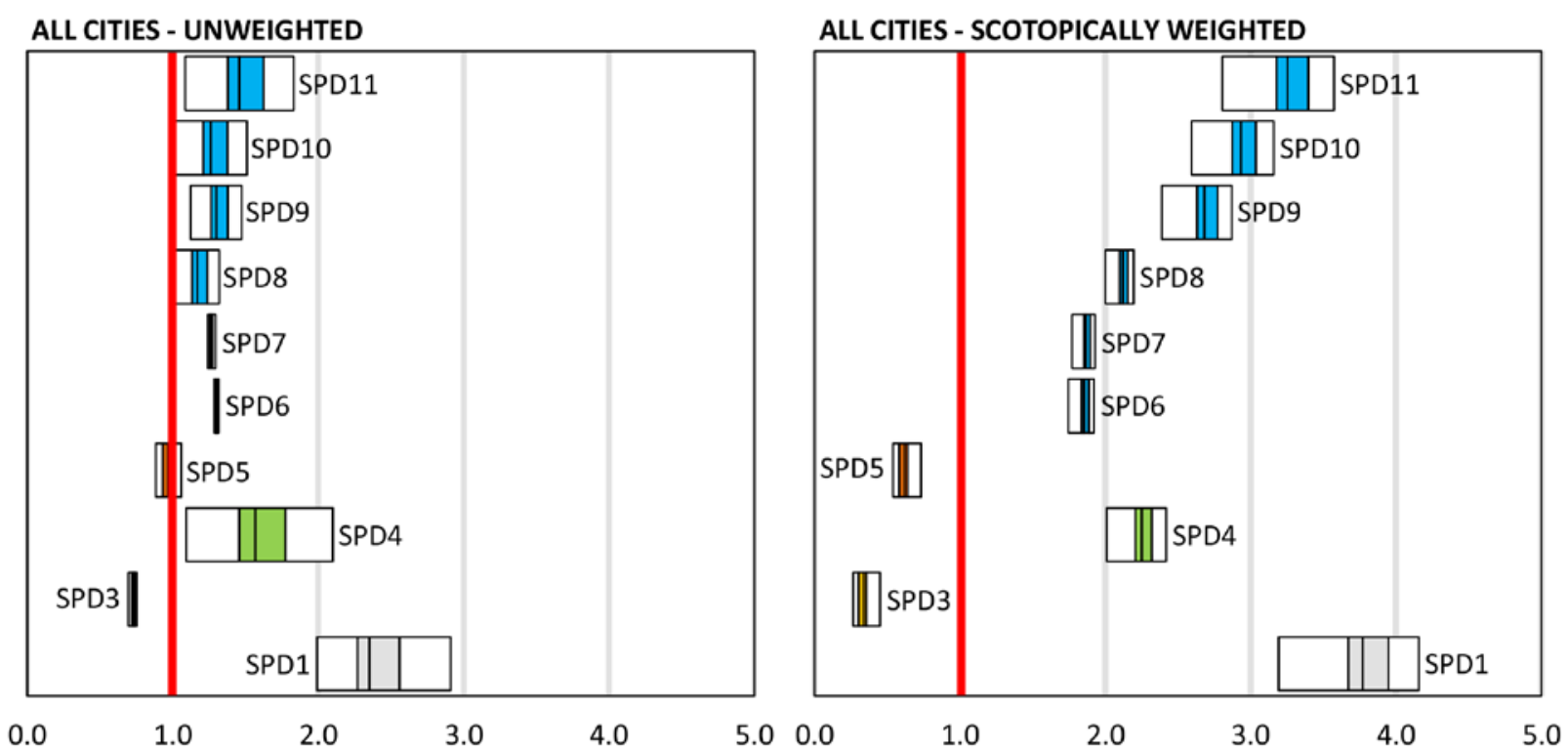

\section{ALL CITIES - UNWEIGHTED - ATM GROUPED}

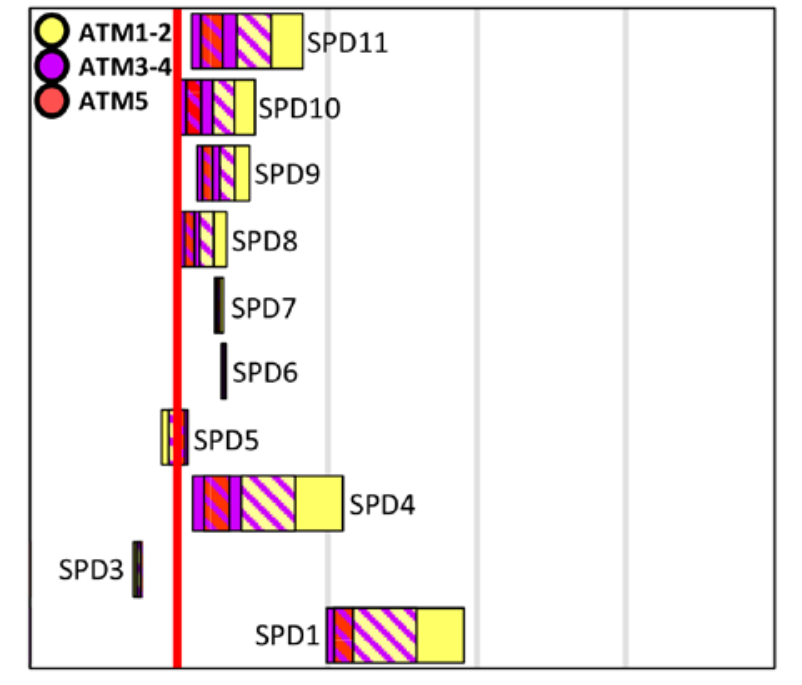

0.0
1.0
2.0

3.0

4.0

5.00 .0

ALL CITIES - SCOTOPICALLY WEIGHTED - ATM GROUPED

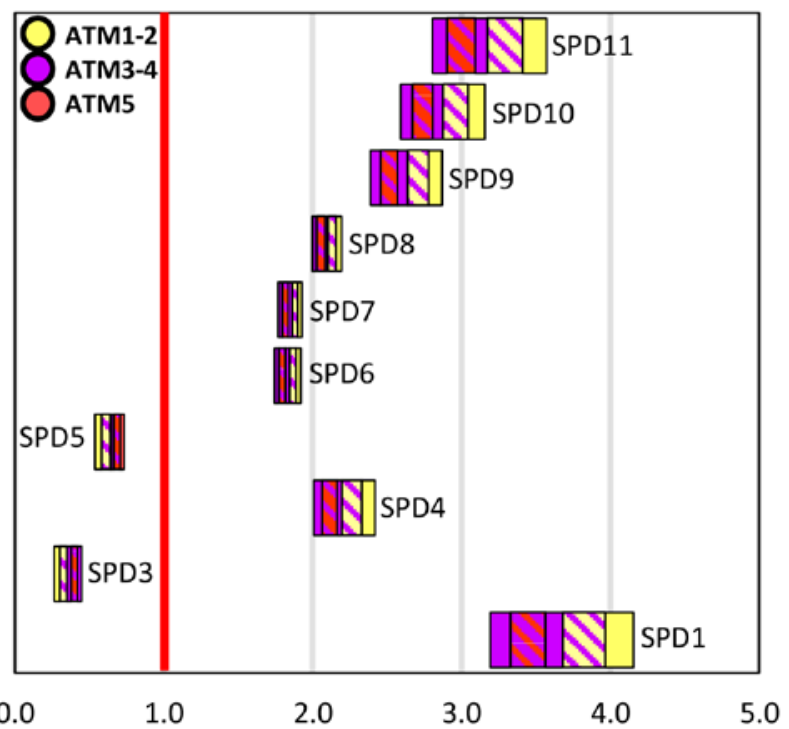

Figure 14 Relative impact of SPD compared to HPS for both observer positions. The top graphs show the minimum, $1^{\text {st }}$ quartile, median, $3^{\text {rd }}$ quartile, and maximum values, with the shaded areas representing data within the $25^{\text {th }}$ to $75^{\text {th }}$ percentiles. Blue indicates white LED sources while red indicates PC Amber LED, yellow LPS, green metal halide, and gray the Equal Energy spectrum. The bottom graphs are split into atmospheric conditions, showing the minimum and maximum values for ATM1-2, ATM3-4, and ATM5. Hatched shading indicates an overlap in atmospheric conditions. Vertical red lines in all graphs represent the HPS baseline at 1.0 . 
The atmosphere more readily scatters shorter wavelengths, so that the color of sky glow under cloudless conditions is likely to be bluer than the color of light emitted from the street lighting (just as with natural sunlight, where the sky appears blue compared to the white light coming from the sun). Figure 15 shows the resulting sky glow spectra for an equal energy source (i.e., an SPD with equal energy emitted at all wavelengths), and thereby represents the relative power of each wavelength in the original source that would be present as sky glow for an observer located near the city. ${ }^{34}$ Notice that, except for ATM5 (the cloudy atmospheric condition), very short wavelengths predominate in the sky glow spectrum for the near observer, followed by blue and decreasing smoothly through the longer colors of the spectrum, with red wavelengths contributing least. Under cloudy conditions, however, wavelengths are scattered more evenly (due to an effect of the attenuation between the source, cloud height, and observer), so there is less spectral variation (and thus the sky or "cloud" glow spectrum appears closer to that of the original source).

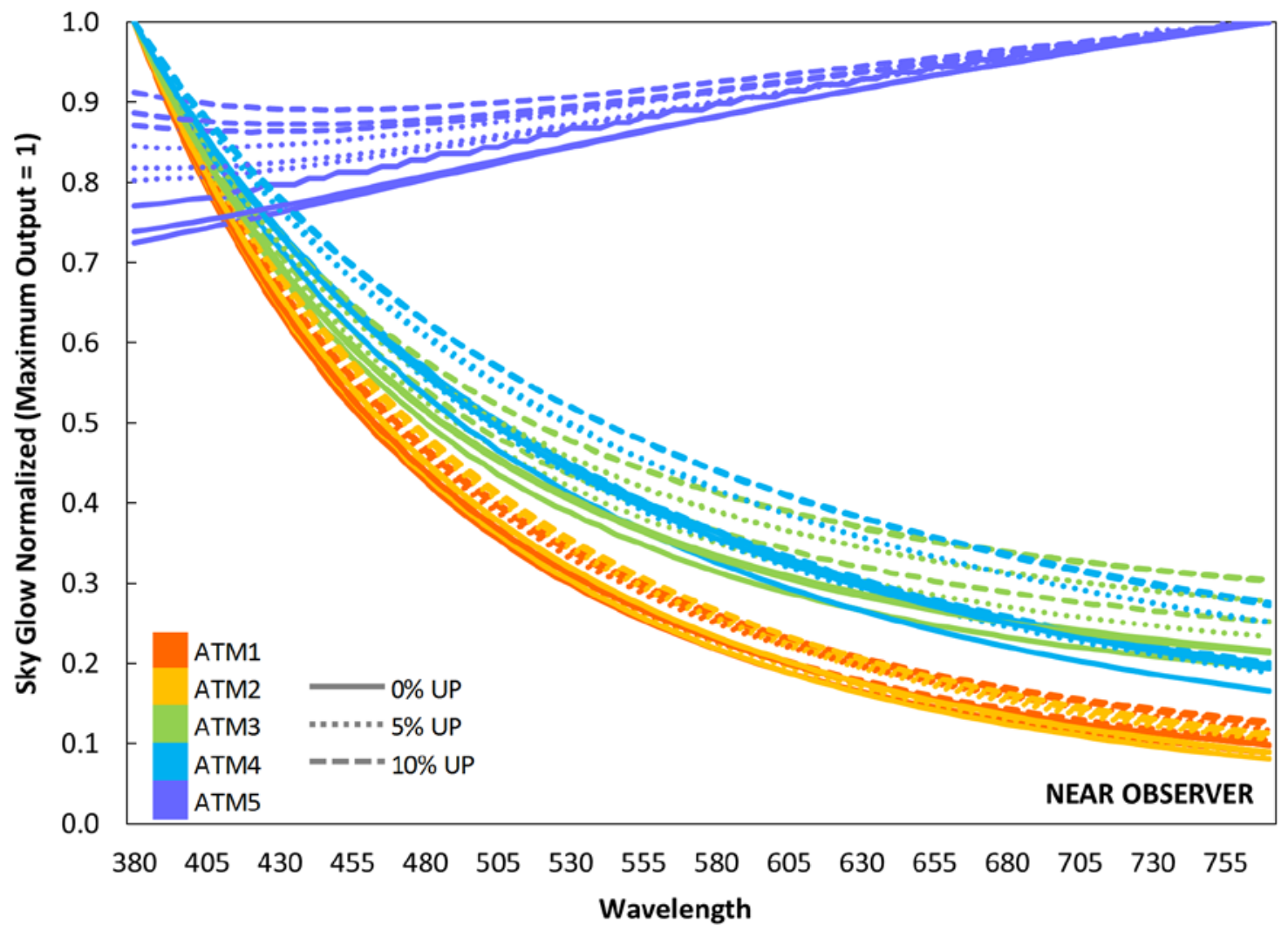

Figure 15 For each city and the near observer position, the relative spectral power distribution of the resulting sky glow for the equal energy SPD based on atmospheric condition and percent uplight.

Shorter wavelengths affect sky glow less for a distant observer than for the nearby observer, because shorter wavelengths are more likely to be scattered or reduced over longer travel distances. This effect becomes even more pronounced when there is cloudiness and turbidity in the atmosphere (Figure 16). While the distant observer in this study is only at $40 \mathrm{~km}$ from the city center, the flattening effect on the SPDs of resulting sky glow from the diminished short wavelength content is already visible.

34 The $2 \%$ uplight runs have been omitted from this graphic for simplicity but fall between the $0 \%$ and $5 \%$ runs. 


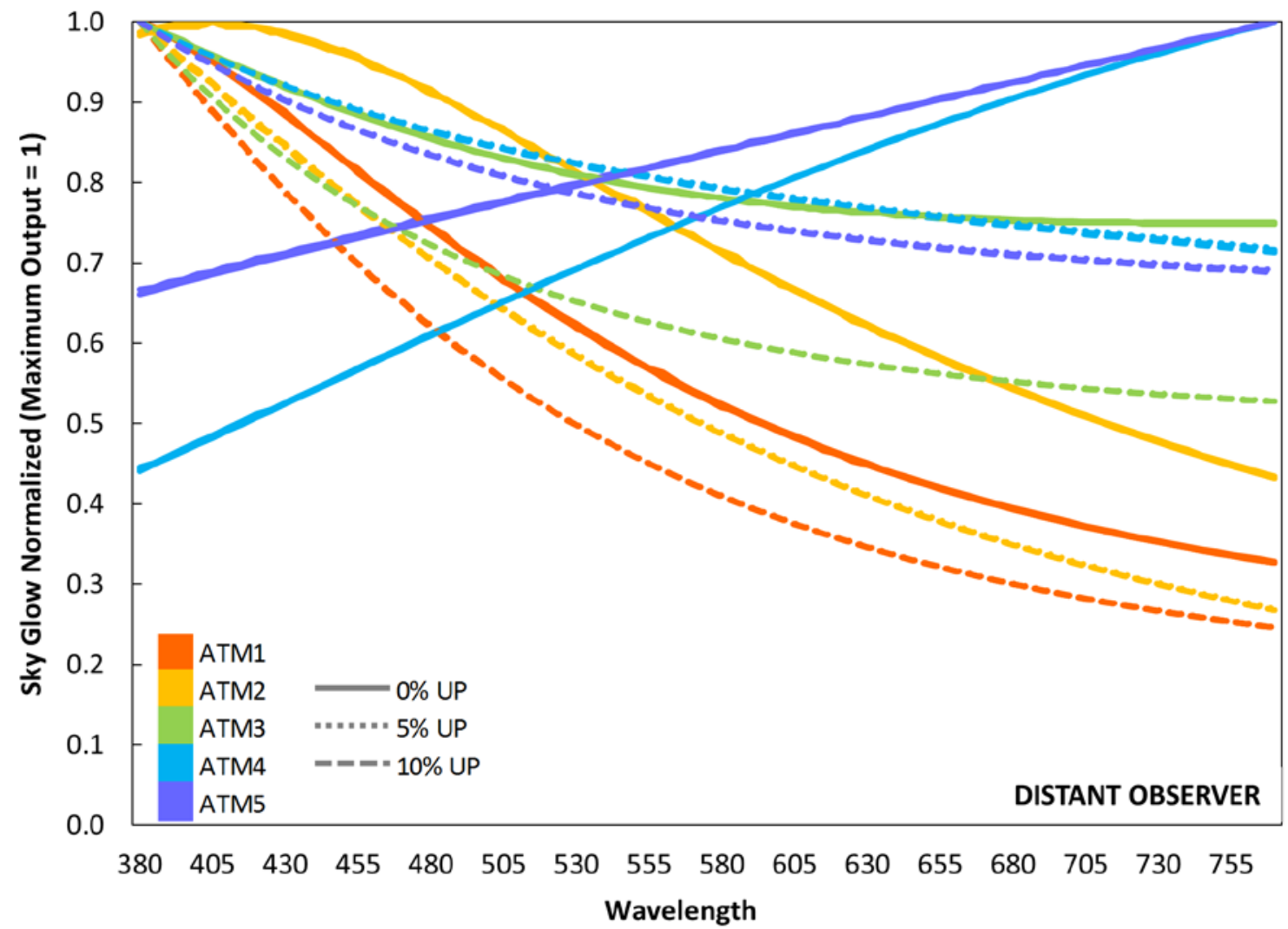

Figure 16 For each city and the distant observer position, the relative spectral power distribution of the resulting sky glow for the equal energy SPD based on atmospheric condition and percent uplight.

At some point (beyond what was modeled here), the shorter wavelengths will be essentially depleted by scattering and will disappear from the SPD of the continuing light. The very turbid and cloudy atmospheric conditions at $40 \mathrm{~km}$ are both already displaying this characteristic because those conditions exert the greatest influence on attenuating short wavelengths near the source, and thus some portion has already been removed by the relatively short distance traveled to the distant observer. Eventually, at some greater distance from the city, all the curves will assume the shape of the two 0\% uplight curves for ATM4 and ATM5 in Figure 16. Note also that even at $40 \mathrm{~km}$ under cloudy conditions, only uplight emitted at low angles above the horizontal plane reaches the distant observer's position, so the magnitude of radiant power at that location has likely been substantially reduced already. 


\section{Discussion of Individual Contributions}

Individual contributions to sky glow from the different variables range widely. Although such contributions do not naturally occur in isolation-in other words the results observed in an actual location reflect the sum total of the individual influences (some of which are counter to others), it is useful to review them separately to better understand the variables (or "levers") a planner has at their disposal for influencing the ultimate impact of a system. Combining these individual contributions is the subject of Section 7.

Figure 17 separates the relative impacts of the variables independent of the lighting system (e.g., atmospheric conditions) from those that can be altered through luminaire selection (e.g., SPD, percent uplight, and light output). In Figure 17, atmospheric conditions are relative to ATM1 and SPD is relative to HPS. The percent uplight values represent a change from $2 \%$ to $0 \%$ uplight and the light output values represent a change from $100 \%$ to $50 \%$ output.

Relative levels of influence are more important in this graph than are the absolute values of the results, since the overall accuracy of the values is only as valid as the assumptions that underlie them (and as noted, the ultimate impact results from the combination of elements). Nevertheless, comparing the relative sizes of corresponding columns provides a useful indication of the different levels of influence.

A number of observations can be drawn from the graphs:

- Atmospheric conditions have the potential to significantly increase the impact on sky glow for near observers.

- Among the characteristics of the street lighting luminaire, SPD has the greatest potential to increase sky glow if a full range of product options (i.e., from LPS to metal halide and all other products in between) is considered; however, when the actual selection considered is between two products relatively close in characteristics, such as a nominal $3000 \mathrm{~K}$ LED versus a $4000 \mathrm{~K} \mathrm{LED}$, the range in impacts is much narrower, even when scotopically weighted.

- As modeled in this study, reducing the percent uplight has the greatest potential to significantly decrease sky glow for observers distant from the city. Figure 17 shows that reducing the uplight from 2\% to $0 \%$ nearly eliminates any increase in sky glow for the distant observer relative to the defined base condition, for all SPDs and lumen output levels.

- While nothing below a 50\% reduction in light output was modeled, it should also be noted that sky glow directly scales up or down with light output for all observer positions. This suggests that, for example, during certain hours when lower light levels might be acceptable for local safety and other criteria, the lighting system could be dimmed to any level (including none) to further decrease corresponding sky glow contributions.

While the graphs do not show the impact of city characteristics, namely city area and lighting density (number of lights per unit area), larger cities and increased lighting density significantly increase the impact for observers inside and distant from the city. This comes as no surprise, given that larger cities tend to generate more light that causes sky glow over greater distances. 

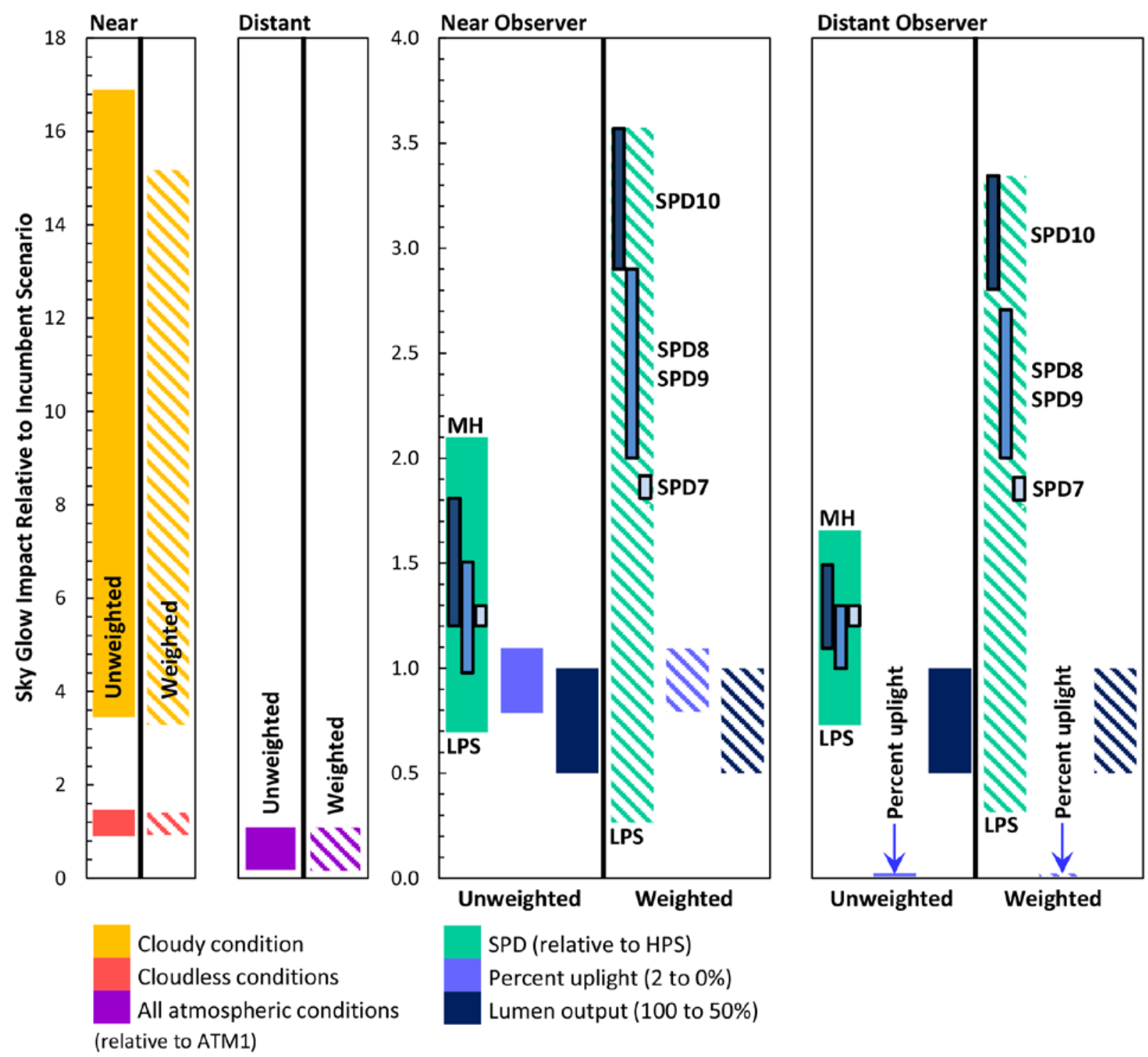

Figure 17 The relative range in impacts of atmospheric conditions (relative to ATM1) on the left, along with SPD (relative to HPS), percent uplight (switching from $2 \%$ to $0 \%$ ), and light output (changing between $100 \%$ and $50 \%$ output) on the right. Solid bars represent unweighted results while hatched bars are scotopically weighted. The specific locations of individual LED SPDs are displayed to facilitate comparisons between them; additional SPDs (e.g., LPS and MH) are included to delineate minimum and maximum performance. 


\section{$7 \quad$ Combined Effects Comparison}

So far, the investigation has focused on the individual influences of different variables on sky glow, whereas the results in an actual conversion come from the particular combination of these elements in a given installation. This section combines the different elements to examine a representative scenario for a typical street lighting conversion in the U.S. today.

The following series of comparisons are in three progressive tiers: the top chart shows the isolated effect of replacing the baseline HPS SPD with the various other LED SPDs modeled with no other modifications; the middle chart adds the effect of reducing luminaire lumen output by half compared to the baseline HPS (a typical result for conversions in the U.S.); and the bottom chart further adds the impact of eliminating uplight from the luminaires, assuming a typical HPS baseline value of $2 \%$. The bottom charts in both figures thereby represent typical conversion scenarios in the U.S.-an incumbent HPS cobra head product with $2 \%$ uplight replaced by each of the LED products listed, at half the light output and $\mathbf{0 \%}$ uplight. Figure 18 and Figure 19 show the three iterations for a near observer and a distant observer, respectively, separated into cloudy and cloudless conditions. The impacts are further divided into the different LED SPDs modeled. While the impacts are clearly evident, a few highlights deserve mention:

- When SPD is the only variable considered (as discussed in the previous section isolating individual impacts), all of the modeled LED conversions other than PC Amber LED increase sky glow relative to HPS.

- Scotopically weighting the results significantly increases the impact compared to unweighted results. Generally, the products with higher levels of short wavelength content have greater impact to sky glow due to Rayleigh scattering effects, and significantly increase that impact as atmospheric turbidity increases, since turbidity intensifies scatter. Scotopically weighting the results further increases the calculated sky glow effect for sources with greater short wavelength content by accounting for the human eye's increased sensitivity to short wavelengths.

- Taking the next step and reducing lumen output by half improves the situation, decreasing unweighted sky glow in all of the LED conversions relative to the baseline HPS system. For the scotopically weighted results, sky glow relative to HPS decreased for some of the LED products but increased for others.

- Ultimately, when both reduced lumen output and percent uplight are taken into consideration, all LED products allow for greater potential reductions at the near observer position, although certain conditions may still increase sky glow overall. In the worst-case scenario modeled in this study (the LED with the most short wavelength content), scotopically-weighting the results increases the contribution to sky glow from street lights about $60 \%$ relative to the HPS baseline, for the near observer position under the highest turbidity. However, even the impacts of this worst case scenario decrease rapidly with distance. For the distant observer (at just $40 \mathrm{~km}$ ), eliminating the uplight dramatically reduces the sky glow for all SPDs, for both the unweighted and scotopically weighted results alike. 

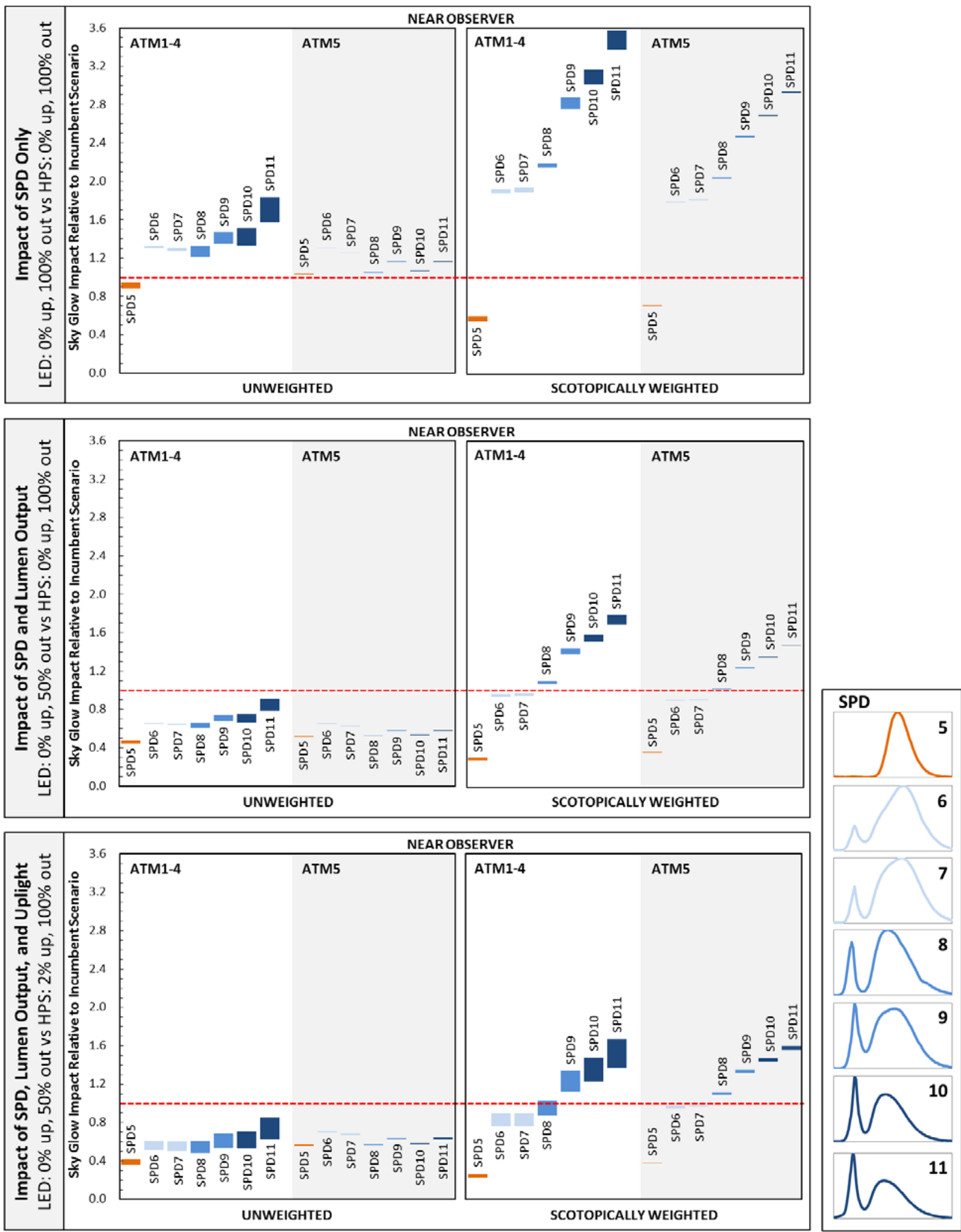

Figure 18 Combined results of the LED street lighting conversions, from the near observer position at the edge of the city. The top chart shows the isolated effect of replacing the baseline HPS SPD with that of the various other SPDs modeled; the middle chart adds the effect of reducing luminaire output by half compared to the baseline HPS; the bottom chart further adds the impact of eliminating uplight from the luminaires, assuming a typical HPS baseline value of $2 \%$ (note this represents an increase from the first two charts using $0 \%$ ). The baseline is represented by the dashed red line at the normalized value of 1.0 . 

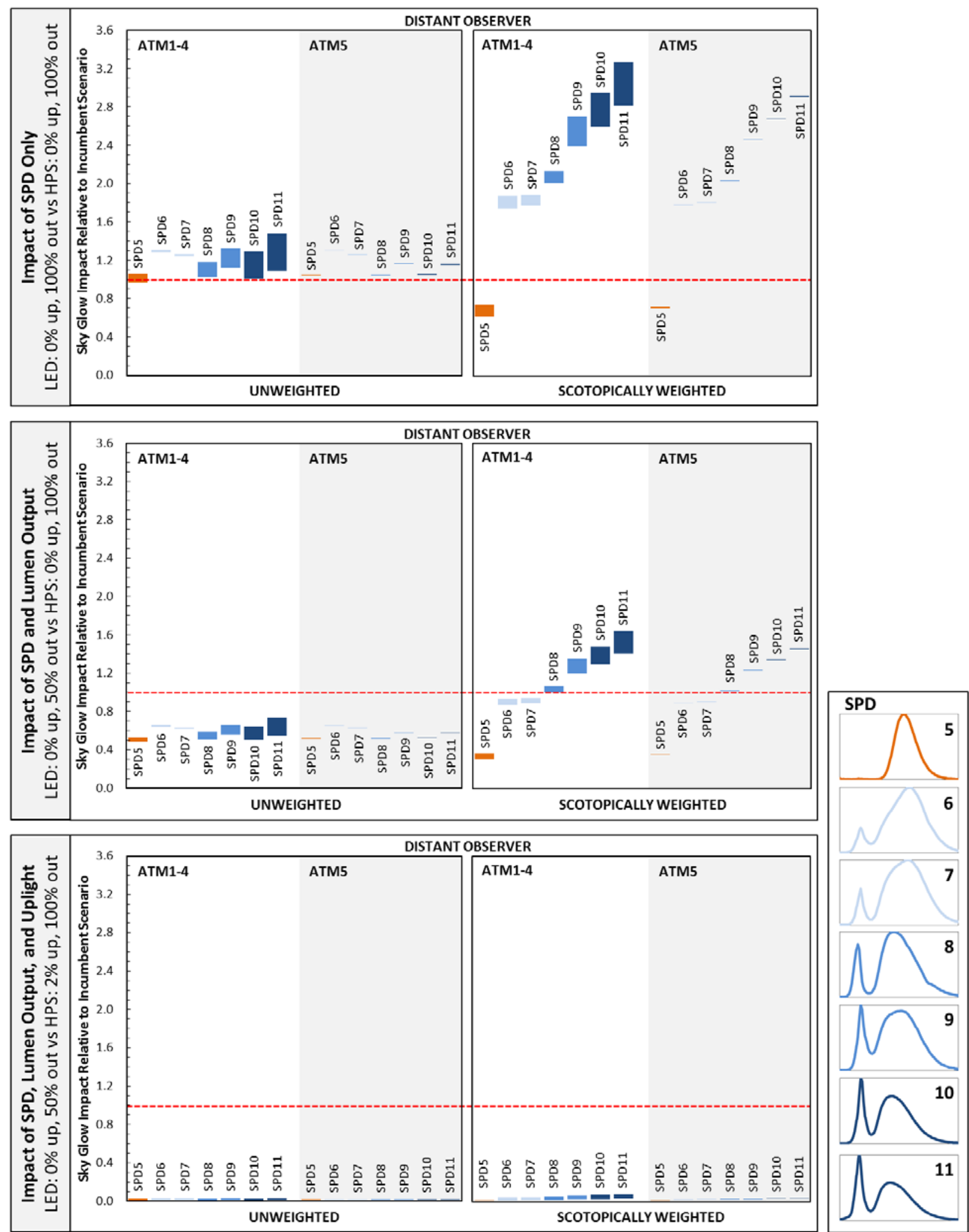

Figure 19 Combined results of the LED street lighting conversions, from the distant observer position $\mathbf{4 0} \mathrm{km}$ from city center. The top chart shows the isolated effect of replacing the baseline HPS SPD with that of the various other SPDs modeled; the middle chart adds the effect of reducing luminaire output by half compared to the baseline HPS; the bottom chart further adds the impact of eliminating uplight from the luminaires, assuming a typical HPS baseline value of $2 \%$ (note this represents an increase from the first two charts using $0 \%$ ). The baseline is represented by the dashed red line at the normalized value of 1.0 . 


\section{$8 \quad$ Takeaways from the Results}

Several useful conclusions can be drawn regarding each contributing element modeled in this study.

\subsection{Light output}

- The relationship between light output and sky glow is linear; increases or decreases in the luminaire output levels are matched one-for-one in the resulting contributions to sky glow from the street lighting system. Light output, and thus target light levels on the ground and how they might be varied both spatially (e.g., between roadway and off-roadway areas) and temporally over the course of an evening, are powerful tools for addressing sky glow and related concerns.

\subsection{Percent uplight}

- For a near observer under clear conditions, a reduction from any level of uplight to $0 \%$ significantly reduces sky glow. For distant observers, sky glow is quickly attenuated at $0 \%$ uplight (although a light dome in the direction of the city may still be visible).

- Under cloudy conditions, the near observer sees more sky glow contributed by reflected downlight than from uplight emitted at small angles above the horizontal plane. This is because the uplight tends to travel out of the area before contributing much sky glow to the immediate area, whereas reflected downlight is essentially trapped in the immediate area between the ground and cloud layer.

- Under clear conditions, transitioning from $10 \%$ to $5 \%$ uplight has less impact than transitioning from $5 \%$ to $0 \%$, signifying that the greatest impact comes from the transition to no uplight.

\subsection{Spectral power distribution}

- Light sources emitting higher levels of short wavelength energy experience more scatter/attenuation in the atmosphere until those wavelengths are depleted. Scotopically weighting the results emphasizes short wavelength content centered on the maximum scotopic sensitivity of $507 \mathrm{~nm}$. Longer wavelengths tend to travel farther and thus have more impact on sky glow for distant observers.

- Higher aerosol contents tend to reduce these differences between short and long wavelengths over distance as they scatter and attenuate wavelengths more uniformly.

\subsection{Atmospheric conditions}

- Cloudy conditions have a large potential impact on sky glow within the defined city area. Clouds reflect, transmit or absorb all wavelengths of light fairly evenly, and the resulting diffusion of the reflected light contributes to the localized sky glow. Clouds eventually prevent any light from reaching a distant observer, although longer wavelengths continue to propagate farther than shorter wavelengths.

- Clear conditions result in more scattering and attenuation of shorter wavelengths than longer ones; for a local observer this means a potentially "bluer" sky glow than the SPD of the original source, but also means that the shorter wavelengths are depleted from a projected beam faster than longer wavelengths. Longer wavelengths thereby affect sky glow more for distant observers, under clear conditions. (As this study only modeled out to a distance of $40 \mathrm{~km}$, the full attenuation of shorter wavelengths does not appear in these results, although the trend is already evident in Figure 16.) 


\subsection{City characteristics}

- In this study, city area and lighting density were the main drivers of total light output and associated sky glow specifically related to characteristics of the city, although this study did not consider complex city geographies (instead simplifying each to a circular layout). Reducing luminaire density by eliminating unnecessary luminaires is one approach that cities might pursue to reduce sky glow.

\subsection{Combined Effects}

- The transition to no uplight nearly removes the contribution of street lighting to sky glow for the distant observer (by at least 95\%), for all products and atmospheric conditions. ${ }^{35}$

- For a near observer, on an unweighted basis, typical conversions to LEDs that include a $50 \%$ reduction in lumen output and elimination of uplight will significantly reduce sky glow compared to an HPS incumbent delivering $2 \%$ uplight. Scotopically weighting the results reduces the number of products that can make this claim.

- Cities can use the knowledge of combined effects to balance street light conversions and new street lighting installations to either maintain current levels of sky glow or reduce the total sky glow over time.

35 In terms of the all-sky horizontal irradiance $\left(\mathrm{W} / \mathrm{m}^{2}\right)$ metric selected for use in this investigation. 


\section{$9 \quad$ Other Considerations}

The preceding sections have been presented without any implied preferences or consideration of additional factors that may bear on the selection of the best characteristics of a street lighting system for a given location. In an actual situation, the choices available to owners and users of a street lighting system often involve a mix of benefits and compromises, of which sky glow is only one. A number of such factors must be weighed by the system designer based on the specific site conditions to find the best balance among them.

\subsection{Energy use}

Until very recently, warm-white LEDs exhibited significantly lower efficacies than cool-white LEDs because of Stokes losses during the phosphor conversion of the blue light output by the LED. In general, the greater the amount of blue light conversion by phosphors required to produce increasingly warmer color temperatures, the greater the associated losses. The difference in efficacy between 3000 and $4000 \mathrm{~K}$ LED luminaires, for example, has been as high as $25 \%$ or more in the past, but is diminishing over time as newer products enter the market. This change is not only a function of improved phosphor materials, but is also due to the development of different approaches that manufacturers can use to alter CCT that may, for instance, preserve efficacy at the expense of some ability to render colors accurately.

Table 5 lists the cutsheet-reported performance of various products that were gathered from manufacturer websites in February 2017. Each row lists a product and its reported performance at the various CCTs in which it was available at that time. The key information in this table is in the final column, which reveals the energy penalty at that time for reducing the color temperature from one value to the next (e.g., from 4000 to $3000 \mathrm{~K}$ or from 5000 to $4000 \mathrm{~K}$ ).

Table 5 Performance specifications, including power (W), light output (Im), and luminaire efficacy (Im/W) of similar make and model products available at different CCTs.

\begin{tabular}{|c|c|c|c|c|c|c|c|c|c|c|c|}
\hline \multirow[b]{2}{*}{ Manufacturer/Product } & \multicolumn{8}{|c|}{ Nominal CCT } & & \multicolumn{2}{|c|}{$\begin{array}{l}\text { Percent Change in } \\
\text { Efficacy from }\end{array}$} \\
\hline & W & $\mathrm{Lm}$ & $\mathrm{Lm} / \mathrm{W}$ & W & $\mathrm{Lm}$ & $\mathrm{Lm} / \mathrm{W}$ & W & $\mathrm{Lm}$ & $\mathrm{Lm} / \mathrm{W}$ & $\begin{array}{c}4000 \mathrm{~K} \text { to } \\
3000 \mathrm{~K}\end{array}$ & $\begin{array}{c}5000 \mathrm{~K} \text { to } \\
4000 \mathrm{~K}\end{array}$ \\
\hline GE Lighting $^{A}$ & 15 & 1900 & 127 & 15 & 2000 & 133 & N/A & $\mathrm{N} / \mathrm{A}$ & $\mathrm{N} / \mathrm{A}$ & $-5 \%$ & $\mathrm{~N} / \mathrm{A}$ \\
\hline Cree $^{B}$ & 50 & 5000 & 100 & 50 & 5000 & 100 & N/A & $\mathrm{N} / \mathrm{A}$ & N/A & $0 \%$ & $\mathrm{~N} / \mathrm{A}$ \\
\hline Eaton $^{\mathrm{c}}$ & 59 & 5414 & 92 & 59 & 6116 & 104 & 59 & 6116 & 104 & $-11 \%$ & $0 \%$ \\
\hline Hubbell $^{\mathrm{D}}$ & 269 & 17734 & 66 & 269 & 22736 & 85 & 269 & 23645 & 88 & $-22 \%$ & $-4 \%$ \\
\hline Leotek $^{\mathrm{E}}$ & 29 & 3451 & 118 & 29 & 3739 & 128 & 29 & 3739 & 128 & $-8 \%$ & $0 \%$ \\
\hline Acuity Brands Lithonia ${ }^{F}$ & 72 & 7137 & $99^{G}$ & 72 & 7665 & 106 & 72 & 7712 & 107 & $-7 \%^{G}$ & $-1 \%$ \\
\hline
\end{tabular}

${ }^{A}$ Evolve ERL1-Type 2 Med

${ }^{B}$ RSW-S1-Type 2 Med

${ }^{\mathrm{C}}$ Galleon LED T2 1000mA

${ }^{D}$ WP9L2P70/120LED

${ }^{\mathrm{E}}$ GCJ1-20H-3-450S-5000K

${ }^{\mathrm{F}}$ DSXO LED $20 \mathrm{C} 1000$ T2M

${ }^{G}$ The manufacturer reports reducing the CRI of the $3000 \mathrm{~K}$ version of this product from 80 to 70 to achieve higher efficacy.

Accurate comparison between products at different CCTs is complicated by the various methods manufacturers use to preserve efficacy, as noted above. However, all such tradeoffs in luminaire characteristics and CCT choices should be evaluated at the time of product selection to optimize the benefits for the specific project. 


\subsection{Visual acuity, scene brightness, and possible safety implications}

Short wavelengths are a part of the natural day and night spectrum around which our vision has evolved.

Reducing the content of short wavelengths may therefore carry tradeoffs in visual performance and perceptions of brightness that need to be considered when designing a street lighting system. The spectrum affects both the luminance contrast and the color contrast of the object, and thus affects the ability to see and recognize the task and situation even when our sensitivity to color is diminished under nighttime light levels.

The source of the visibility and brightness improvement from broader-band spectrum sources is not completely understood. The $\mathrm{IES}^{36}$ and the $\mathrm{CIE}^{37}$ acknowledge improvement in off-axis visibility for drivers due to scotopic content in the SPD, probably due to the blue-shifted sensitivity of the rods and the preponderance of rods in the periphery of the retina, but more research will be required to fully explain this phenomenon.

Studies in Seattle ${ }^{38}$ and San Jose ${ }^{39}$ suggest that color contrast may play a role in a driver's ability to detect an obstacle or pedestrian from a greater distance, enabling earlier braking time. Although more research is again needed, it stands to reason that there may be associated safety implications from reducing or eliminating short wavelengths from a street lighting source.

\subsection{Sky glow from street lighting in context}

Sky glow results from a complex combination of light released to the exterior environment within a region. In addition to street lighting, other sources of light at night include area lighting (e.g., surface parking lots and parking garages, pedestrian pathways and building campuses, recreational parks and athletic fields, and residential porch lights), advertisements and signage, vehicles, exterior building architectural lighting, and building interior lighting escaping from windows and skylights (see Figure 20).
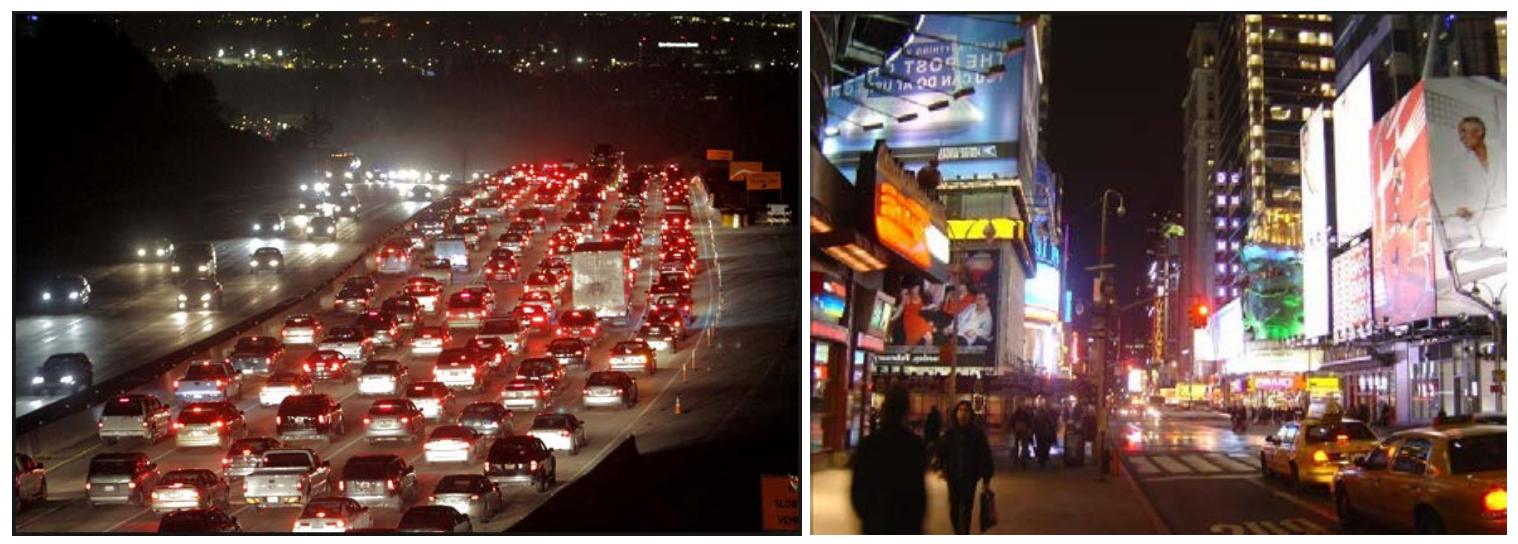

Figure 20 Many sources of light contribute to sky glow.

While a street light would typically have a much higher light output than, for instance, light escaping a single office or storefront window, the uplight component of the latter may be greater due to a complete lack of directional control. Furthermore, the aggregate contribution may be quite substantial, as suggested in Figure 21.

36 Illuminating Engineering Society. Spectral effects of lighting on visual performance at mesopic light levels, TM-12, 2006.

37 Commission Internationale de L'Eclairage, "Recommended System for Mesopic Photometry Based on Visual Performance," CIE Report (Vienna Austria), vol. 191, 2010.

38 Seattle LED Adaptive Lighting Study, 2014: https://neea.org/docs/default-source/reports/seattle-led-adaptive-lightingstudy. pdf?sfvrsn=4.

39 City of San Jose Advanced street lighting technologies assessment project, 2010:

http://www.sanjoseca.gov/DocumentCenter/View/18941. 
Building lighting is predominately characterized by broad spectrum sources at $3000 \mathrm{~K}$, and frequently higher, CCTs. Associated sky glow is thereby likely to be significant, but there appears to be a relative lack of focus on this source and its corresponding contributions to date.

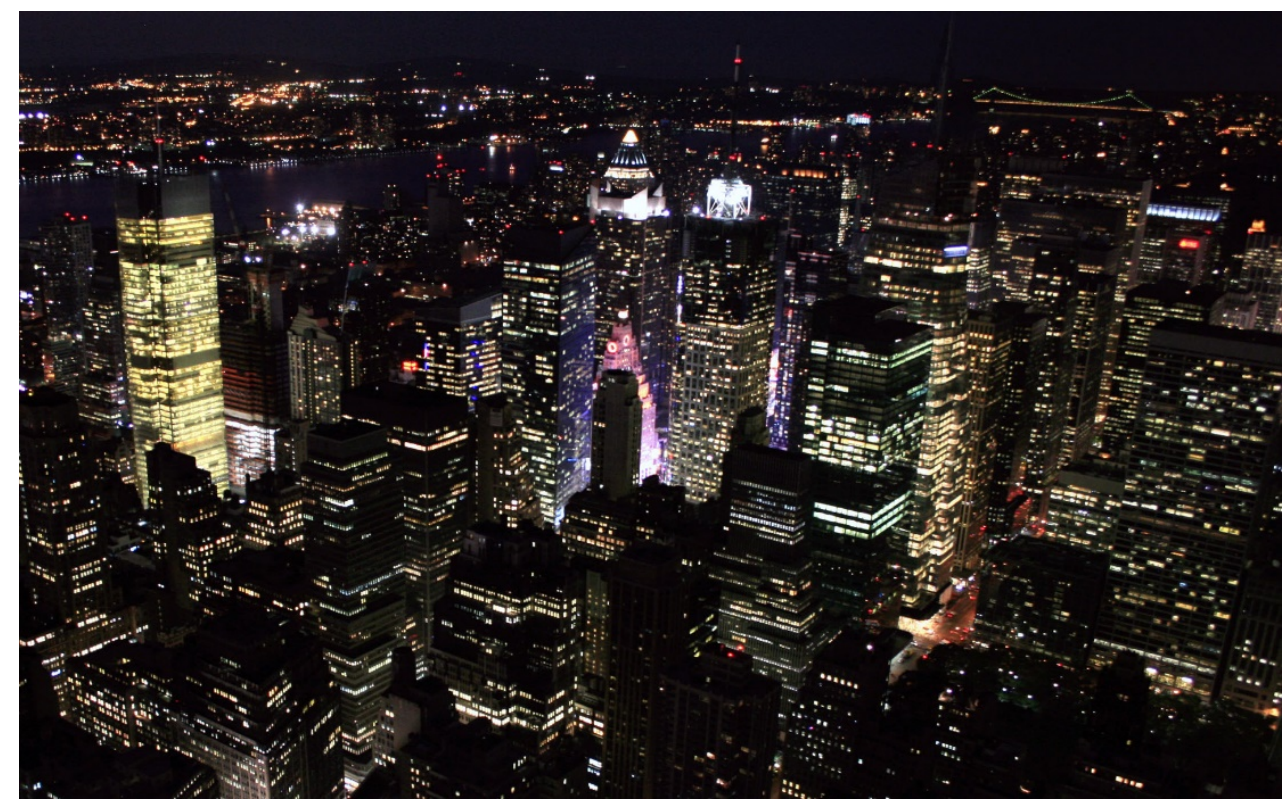

Figure 21 High-angle lighting primarily from building interiors. All light visible from this point of view is being emitted above the horizontal plane.

Table 6 was assembled in response to recent concerns from the medical community about "blue" wavelength content in LED sources, with a focus on street lighting. ${ }^{40}$ The table reveals that blue wavelength content is generally similar among different lighting sources at similar CCTs (though individual products can range somewhat due to differences in their SPDs). Note that the sources listed include many commonly used in interior and exterior applications. All broad-spectrum lighting exposed to the night environment thus has the potential to be of concern, with respect to contributions to sky glow.

The model runs described in this study illustrated that uplight exerts an oversized influence on sky glow and that the influence generally increases with SPDs containing higher levels of short wavelength energy, especially if weighting the results for scotopic visibility. Heavily populated metro areas thereby have potentially substantial contributions to sky glow from non-street lighting sources. This is emphasized following LED lighting conversions where direct uplight from the older street lighting has been largely eliminated. Estimation of the relative contributions of non-street lighting sources has received only limited attention in the literature to date, however, and deserves further investigation.

Ultimately, cities should proceed through their street lighting design and selection process with as much knowledge as possible of the benefits and trade-offs of the different options available in order to have realistic expectations of the results. This recommendation holds whether the topic of interest is energy use, lighting quality, associated issues like sky glow, or achieving the best balance among all of the above.

40 See the related Light Post newsletter: http://energy.gov/sites/prod/files/2016/07/f33/msslc_enews_jul2016.pdf. 
Table 6 Selected blue light characteristics of various outdoor lighting sources at equivalent light output.

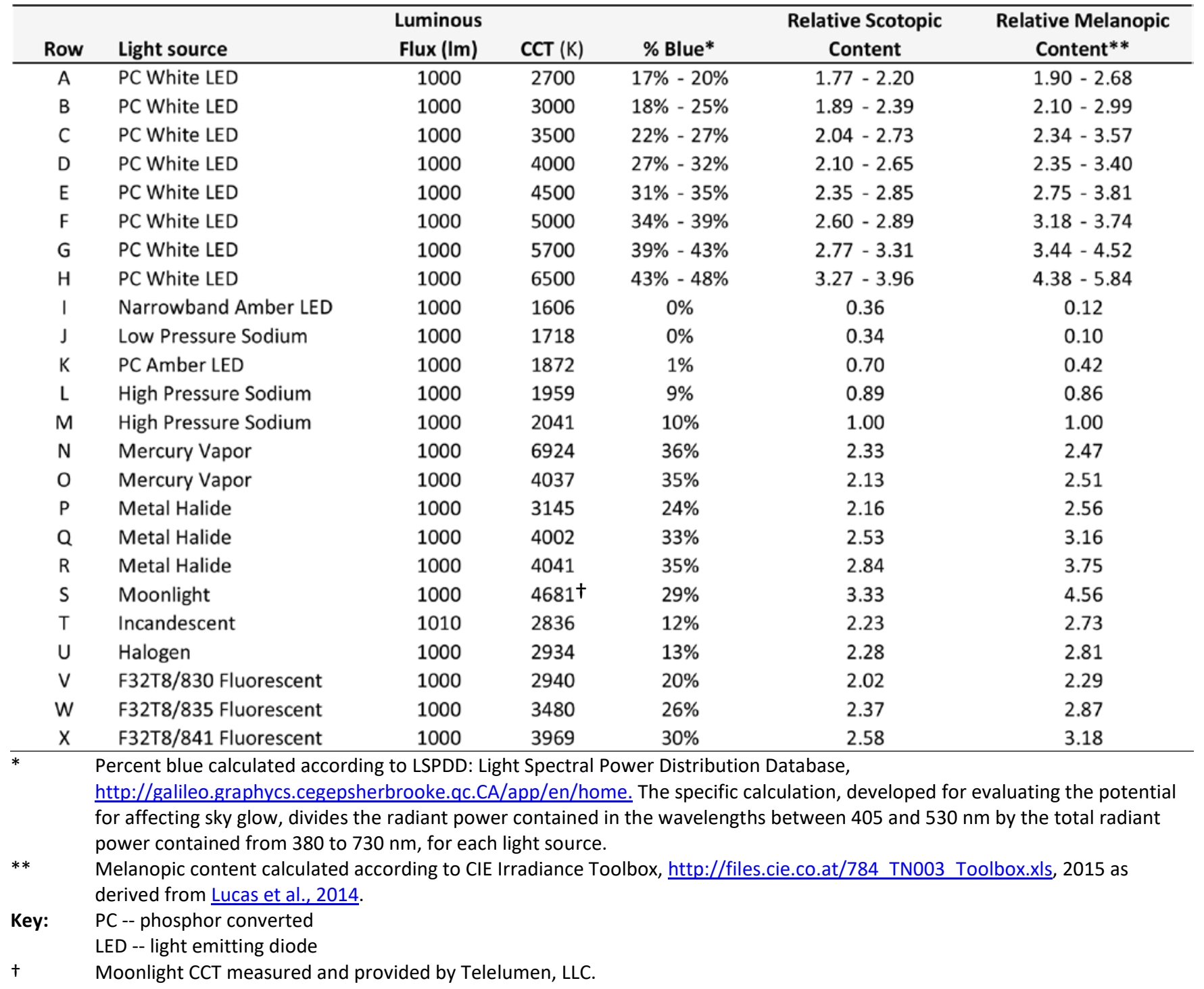




\section{Conclusions}

Multiple factors contribute to the level of sky glow produced in any location, and the level likely changes with the combination of those factors (and sometimes dynamically as factors such as weather vary over time). A related consideration is that street lighting is only one of many sources of light at night in urban areas. The findings in this study consequently represent only the estimated contributions to sky glow from the street lighting system under the conditions considered, and may not even address the primary sources of sky glow contribution in locations like large urban areas.

The short wavelength content of broad-spectrum sources like LEDs is only one element within the street lighting system. Other characteristics of modern street lighting luminaires can reduce or, in some cases, even eliminate the effects of wavelength content on sky glow. The three main characteristics of luminaires that influence sky glow are SPD, total light output, and light distribution (and, most importantly, the amount emitted as uplight above the horizontal plane). Each of these characteristics can be varied during at least the initial selection of products and should therefore be carefully evaluated as part of the system design.

External factors also influence the relative amount of sky glow that a given observer receives, such as the observer's location relative to the source of the light (especially their distance from it), the size of the source (i.e., city size), and the atmospheric conditions at the time. These points are of interest but may or may not be of value to someone planning an installation since they are largely outside of the range of influence of those plans.

This investigation looked at relative sky glow values produced by varying one factor at a time, so that the effect of SPD, for example, can be compared between sources with all other factors being equal. This investigation also looked at relative sky glow for a typical LED streetlight retrofit in the U.S., where lumen output is reduced by half, and uplight from a glass refractor HPS cobra head is replaced with a luminaire directing light downward only. The results of this common scenario show that, for residents near the city, the visible contribution to sky glow from a typical LED street light conversion in the U.S. ${ }^{41}$ should be no worse than before, and may possibly improve, relative to the system being replaced. In terms of impact specific to astronomical observation, the overall contribution to sky glow from the street light system should be considerably reduced (by perhaps more than half) for any observatories near the city. Within some relatively short distance outside the city, the sky glow contribution from this typical street light system conversion appears to substantially diminish at all SPDs (and CCTs), for all observers.

Compared to the $4000 \mathrm{~K}$ street lighting systems that much of the country has installed to date, additional reductions in the contribution to sky glow can be pursued through approaches such as adding a dimming system, eliminating unneeded street lights, or moving to a $3000 \mathrm{~K}$ (or lower CCT) standard. However, in this latter case, the specific SPDs of the products under consideration must be examined to confirm that a reduction is being achieved. The merits of doing so should be evaluated case by case, carefully comparing the additional collective benefits with any tradeoffs.

41 A typical street lighting conversion is here defined to mean the replacement of HPS fixtures with $2 \%$ uplight with LED products at half the light output and $0 \%$ uplight. 


\section{Appendix A: Sky Glow Models Reviewed}

The modeling of proposed changes in ground-based light sources could be very helpful in designing optimum parameters of outdoor lighting for a given location, if the model's use is straightforward enough for members of the general lighting community. Numerous models exist to estimate sky glow by approximating the distribution of scattered light, but they vary in complexity and transparency of operation. ${ }^{1}$ The selection of a model depends on the accuracy required as well as the size of the input data and sensitivity to physical parameters.

This section briefly compares three sky glow models developed to date.

\section{The Garstang model}

Originally developed in the 1980s and designed to execute on an Apple II computer of that era, the methodology used in this model has become a standard reference for subsequent models. ${ }^{2}$ Garstang's approach is typically used to predict the effects of outdoor lighting on sky brightness for an observer some distance from the city center. Along a given path through the atmosphere, the model estimates the amount of light scattered, as well as the amount removed by extinction (i.e., attenuation via both scattering and absorption). Garstang's model assumes all light is emitted at $550 \mathrm{~nm}$. A two-component atmosphere is modeled, consisting of molecules and aerosols, with the amount of aerosol defined through a parameter $\mathrm{K}$, which sets the ratio of total molecular (Rayleigh) to aerosol (Mie) scattering. It has been used to predict and evaluate the effects of outdoor lighting on sky glow at astronomical observatories.

The Garstang model was developed using measures of sky brightness to deduce unknown characteristics of onthe-ground lighting - a sky-down approach. It predicts sky glow based on assumed ground lighting characteristics, including total lumen output based on population estimates and assumed lumens per capita, the fraction of light emitted above horizontal (10\% uplight), and the average ground albedo. The model assumes the uplight is emitted close to horizontal plane and the reflected component from the ground is reflected in a Lambertian distribution. Together, they form a composite street lighting distribution. The model addresses the radiative transfer portion of the problem through molecular and aerosol scattering and absorption, has provisions for absorptive (haze) layers, and has differing altitudes between light sources (e.g., cities) and corresponding observation points.

\section{Luginbuhl's modifications to Garstang's model}

Luginbuhl reported that "[t]he standard Garstang model...clearly predicts a much brighter sky than that measured." ${ }^{3}$ Several factors likely contribute to this observation. Garstang assumed there is no atmosphere between light fixtures and the ground; thus, all downward-directed lighting is undiminished by atmospheric

1 For example, a number of examples can be found by searching the internet on the phrase "sky glow model" plus the following additions: Garstang 1991, Cinzano et al. 2000, Gillet et al. 2001, Aube et al. 2005, Baddiley 2007, Kocifaj 2007, Luginbuhl et al. 2009, Kocifaj 2010, Kocifaj et al. 2010, Cinzano and Falchi 2012, Kocifaj et al. 2014, Luginbuhl et al. 2014, Aube 2015, Kollath et al. 2016. R. H. Garstang. 'Model for Artificial Night-Sky Illumination.' Publications of the Astronomical Society of the Pacific, vol. 98, no. 601, 1986, pp. 364-375.

3 C. Luginbuhl et al. 'From the Ground Up II: Sky Glow and Near-Ground Artificial Light Propagation in Flagstaff, Arizona.' Publications of the Astronomical Society of the Pacific, vol. 121, 2009, pp. 204-212. 
scattering or absorption. Also, all ground near the sources is assumed flat and horizontal. ${ }^{4}$ The model furthermore assumes a fixed fraction of the flux is emitted upward, which is increasingly out of date with the expanded use of modern $U 0$ rated luminaires. ${ }^{5}$ All rays reflected from the ground or emitted from fixtures directly upward propagate into the atmosphere, unimpeded by further interaction with surfaces. Thus, the upward intensity distribution function assumed by Garstang (Figure A.1, left) and similar functions, heavily weighted toward the horizon, are unlikely to represent the actual upward light distribution in most cities.
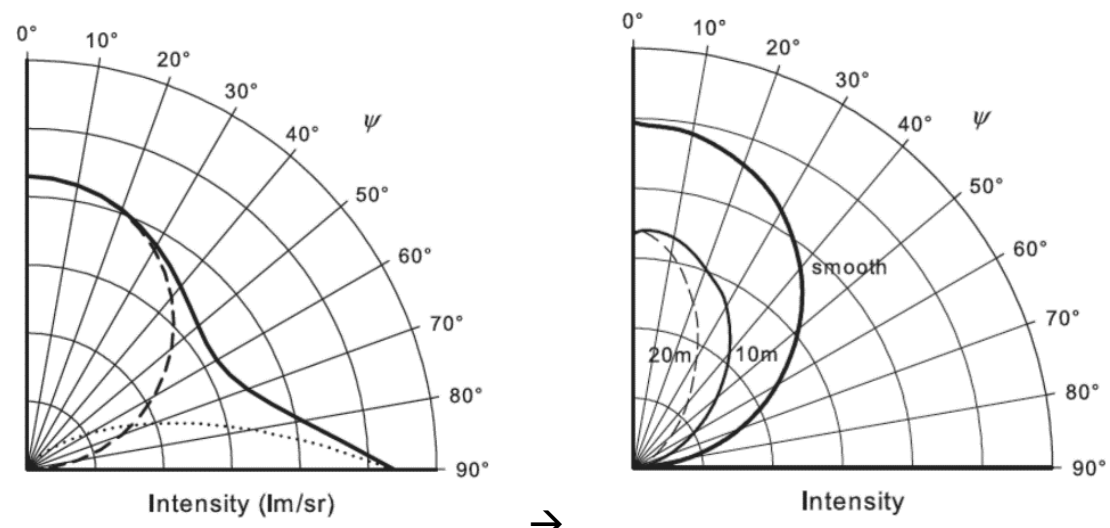

Figure A.1 Mapped upward intensity distribution of Garstang's model (left) compared with Luginbuhl's modifications (right). Left: reflected component (15\%) of the downward-emitted light (dashed line) along with the direct upward component (10\%) (dotted line) together yield upward intensity angular distribution (solid line). Right: smooth earth simulation (solid line) compared with intensity distribution when light fixture is immersed in an array of buildings (inner curves).

Luginbuhl investigated the effects of the near-ground environment by developing specific built environments that accounted for light distribution from specific fixtures, and then studied the resulting impact on the propagation of light (Figure A.1, right). Near-ground extinction is also incorporated through a combination of the direct blocking of some fraction of the near-ground light so the emitted light never reaches the ground and subsequent blocking of reflected rays that consequently never reach the atmosphere. Luginbuhl's follow-up work looked at the effect of light source spectral power distribution (SPD) on sky brightness so as to no longer assume monochromatic radiation. ${ }^{6}$

\section{ILLUMINA ${ }^{7}$}

The difficulty of predicting atmospheric behavior with acceptable precision is well recognized in the field of weather forecasting. Similarly, although a simple sky glow model may provide a cursory estimate of light scatter and propagation in the atmosphere, a supercomputer is usually required to process large amounts of data to help increase confidence in the predictions. For the scope of this paper, Garstang's model is considered "simple" and ILLUMINA the opposite.

4 In reality, if a portion of the light exiting the fixture strikes a surface oriented perpendicular to ground (e.g., the side of a building), no more than $50 \%$ of reflected light will be directed upward, which most heavily affects rays directed close to horizontal.

5 Illuminating Engineering Society Technical Memorandum 15-2011, Luminaire classification system for outdoor luminaires.

6 C. Luginbuhl, P. Boley, and D. Davis. 'The impact of light source spectral power distribution on sky glow.' Journal of Quantitative Spectroscopy \& Radiative Transfer, vol. 139, 2014, pp. 21-26.

7 Wiki de Martin Aube - ILLUMINA project: Heterogeneous modeling of artificial sky radiance. Project page: http://cegepsherbrooke.qc.ca/ aubema/index.php/Prof/IllumEn Read me: https://bitbucket.org/aubema/illumina User's guide: http://cegepsherbrooke.qc.ca/ aubema/index.php/Prof/IlluminaGuide2016 
ILLUMINA (developed by Martin Aubé) is an open-source, voxel-based, radiative flux transfer program that can require weeks to run on a supercomputer with several thousand CPUs and terabytes of RAM. ${ }^{8,9}$ The model is currently housed at Cégep de Sherbrooke in Canada. Although Garstang's model includes an ad hoc term for double scattering, it is not possible to determine whether it correctly models the atmospheric optics. Instead of an ad hoc term, ILLUMINA considers diffuse reflections from the ground and in-scattering of scattered light from volumes $m$ into the volumes $n$ visible to the observer (see Figure A.2).

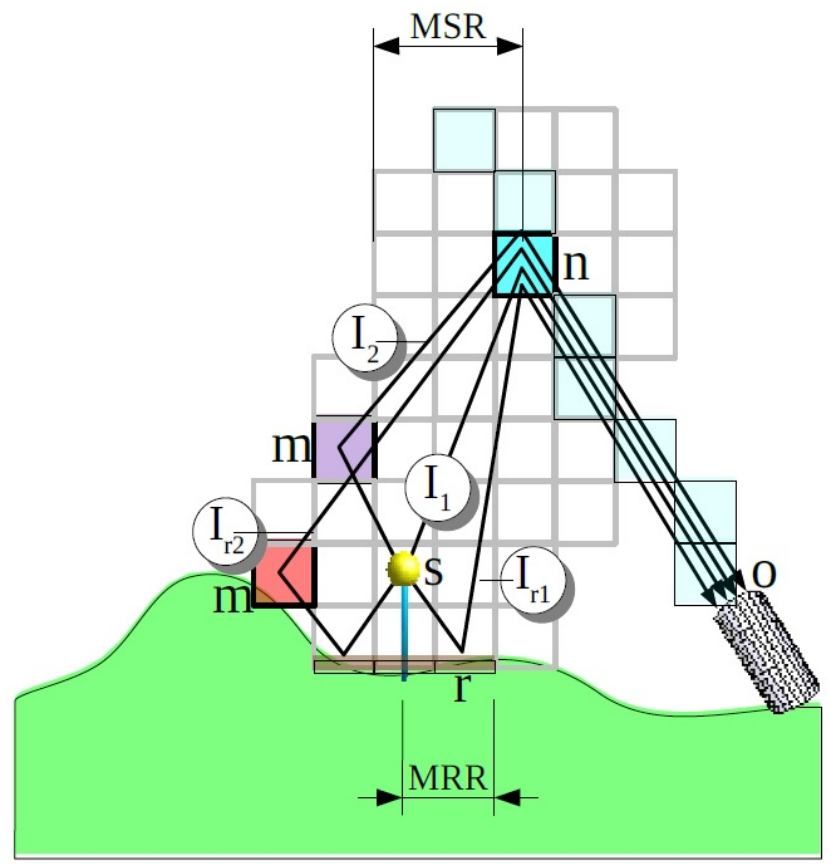

Figure A.2 ILLUMINA considers diffuse reflections from the ground and in-scattering of scattered light from volumes $m$ into the volumes $\mathrm{n}$ visible to the observer. This graphic shows the contribution of the received flux by a spectrometer in position 0 . The animation in the following link shows the 3D calculation of single and double scattering with or without reflection on the ground: http://cegepsherbrooke.qc.ca/ aubema/index.php/Prof/IllumEn?action=download\&upname=illum-anim.gif.

Aubé decided to implement a heterogeneous sky glow model that allows the simulation of real situations (e.g., a city of any shape, geographical distribution of light, spectral luminosity and angular emission patterns, ground spectral reflectance variability, and topography. Remotely sensed satellite data is used to determine the terrestrial information, such as luminosity data, ground albedo, and topography instead of assuming a population density to light intensity relationship as is the case in most models. ILLUMINA was designed to simulate light scattered back to a spectrometer. The approach is similar to ray-tracing software: essentially a set of photons emitted from luminaires in combination with respective interactions with the ground, molecules, and aerosols. These interactions are computed along light-paths toward a simulated observer for a given voxel intersecting a viewing angle (composed of zenith and azimuth angles). A spectrometer was subsequently designed so it could be used to validate model results or simply monitor sky glow for specific sites. The model can also be used independently to simulate the fraction of sky glow due to the molecules and atmospheric aerosols, based on the assumption that the composition and vertical profile of aerosols are horizontally uniform over the modeling domain.

8 M. Aubé et al. 'Light Pollution Modelling and Detection in a Heterogeneous Environment: Toward a Night Time Aerosol Optical Depth Retrieval Method.' Proceedings of SPIE, vol. 5890, no. 248, 2005.

9 M. Aubé. 'Light Pollution Modeling and Detection in a Heterogeneous Environment.' STARLIGHT Proceedings, 2007, pp. 119-126. 
ILLUMINA also gives two maps as standard output: 1) a radiance contribution map (RCM) that provides the contribution of each squared kilometer sea-level footprint of the domain to the sky radiance and 2) a radiance per lumen sensitivity map (RSM) that provides the contribution of each squared kilometer sea-level footprint of the domain to the sky radiance per lumen installed. RCM allows for the identification of the origin of the radiance whereas RSM gives a map of the most critical zones in terms of possible increases or decreases of radiance with any change in light-fixture inventory. 

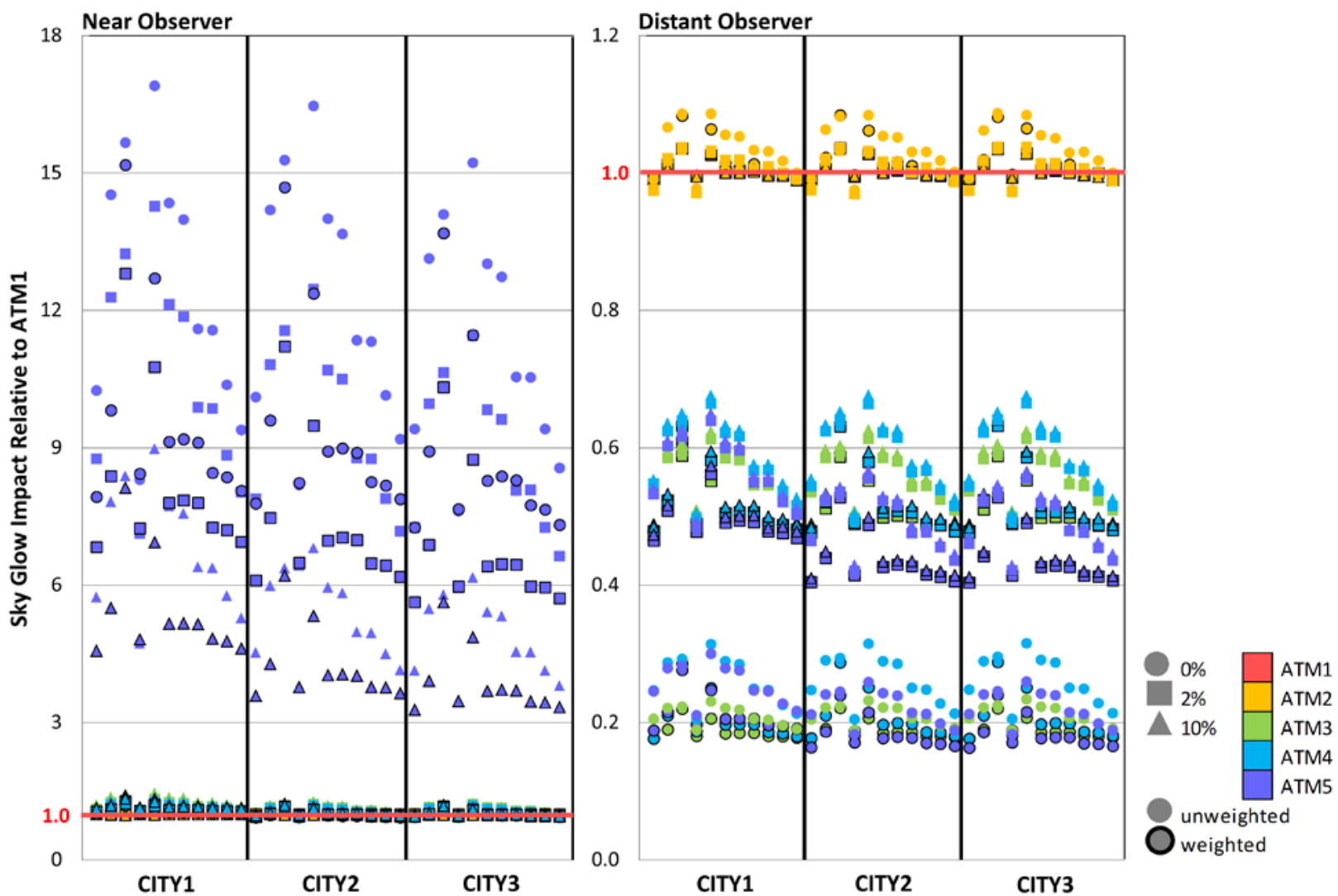

Figure B.1 Relative impacts of the atmospheric conditions for the near and distant observer positions. The relative impacts of all atmospheric conditions compared to ATM1 are graphed for each city and all SPDs based on their percent uplight (0\%, $2 \%$, $10 \%)$. Both the unweighted and scotopically weighted results are graphed. The graph on the left, for the near observer, shows the significant increase in sky glow due to clouds, including the increase in sky glow that comes from decreasing uplight (for the cloudy condition only). The graph on the right, for the distant observer, shows the influence more turbulent and cloudy atmospheres have to decrease sky glow. Unlike the graph for the near observer, decreasing the percent uplight decreases sky glow, also denoted by the arrows. 